\title{
SMALL-STENCIL 3D SCHEMES FOR DIFFUSIVE FLOWS IN POROUS MEDIA
}

\author{
Robert Eymard ${ }^{1}$, Cindy Guichard ${ }^{2}$ And RAPhà̀le Herbin ${ }^{3}$
}

\begin{abstract}
In this paper, we study some discretization schemes for diffusive flows in heterogeneous anisotropic porous media. We first introduce the notion of gradient scheme, and show that several existing schemes fall into this framework. Then, we construct two new gradient schemes which have the advantage of a small stencil. Numerical results obtained for real reservoir meshes show the efficiency of the new schemes, compared to existing ones.
\end{abstract}

Mathematics Subject Classification. 65N30,65N08, 76S05

Received November 28, 2010. Revised April 25, 2011.

Published online October 12, 2011.

\section{INTRODUCTION}

Underground engineering simulations often involve computations of the flow on meshes that are adapted to the geological layers. This geology fitting is likely to produce a number of complex geometrical features, such as faults, vanishing layers, slanted wells, highly heterogeneous permeability fields... The resulting grid cells may feature non-planar faces and they are defined in most cases by 8 vertices; they can be locally defined by 7,6 or 5 vertices for layers which are not present over the whole domain. These grid cells are often called generalised hexahedra. For instance Figure 1 presents a typical near-well mesh used in reservoir engineering; the mesh on the right features distorted cells, high contrasted sizes and non-conforming connections between the radial part (on the left) and the Cartesian part.

Since most of the coupled phenomena which occur in underground flow involve diffusion terms, we focus in this paper on the following simple diffusion problem posed on an open bounded subset $\Omega \subset \mathbb{R}^{3}$, with boundary $\partial \Omega=\bar{\Omega} \backslash \Omega$ :

$$
\left\{\begin{array}{l}
-\operatorname{div}(\Lambda(\boldsymbol{x}) \nabla \bar{u})=f \text { in } \Omega \\
\bar{u}=0 \text { on } \partial \Omega
\end{array}\right.
$$

where $\bar{u}$ is an unknown field (temperature, pressure, $\ldots), \Lambda(\boldsymbol{x})$ is a linear operator which is usually self-adjoint, and with eigenvalues belonging to $[\underline{\lambda}, \bar{\lambda}], 0<\underline{\lambda} \leq \bar{\lambda}$, and $f \in L^{2}(\Omega)$ is a volumetric source term.

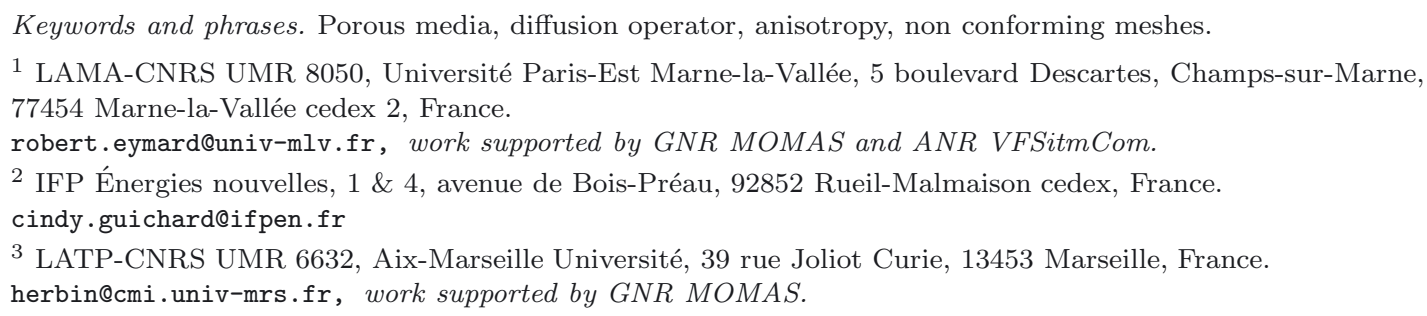



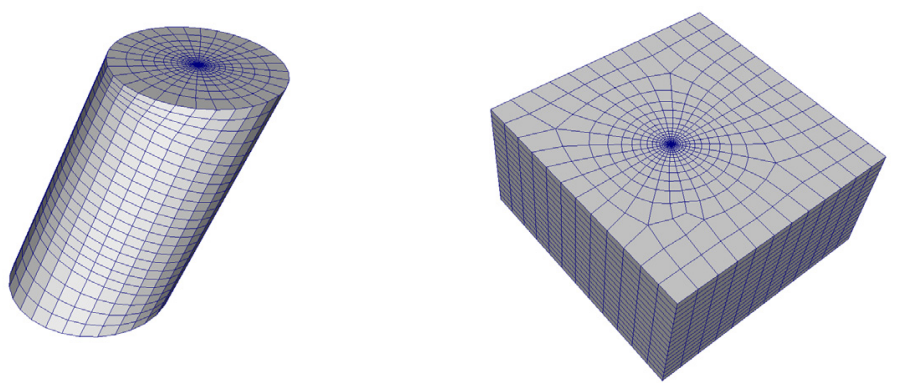

FiguRE 1. Near-well grid: radial mesh without (left) and with transition zone (right).

Hence we wish to approximate the function $\bar{u}$ solution of the weak form of the problem, that is:

$$
\bar{u} \in H_{0}^{1}(\Omega) \text { and } \forall \bar{v} \in H_{0}^{1}(\Omega), \int_{\Omega} \Lambda(\boldsymbol{x}) \nabla \bar{u}(\boldsymbol{x}) \cdot \nabla \bar{v}(\boldsymbol{x}) \mathrm{d} \boldsymbol{x}=\int_{\Omega} f(\boldsymbol{x}) \bar{v}(\boldsymbol{x}) \mathrm{d} \boldsymbol{x} .
$$

The discretization of Problem (1.1) by a finite volume method requires the approximation of the diffusion flux $\bar{\Phi}_{K, \sigma}(\bar{u})$ through any face $\sigma$ of a control volume $K$, defined by $\bar{\Phi}_{K, \sigma}(\bar{u})=-\int_{\sigma} \Lambda(\boldsymbol{x}) \nabla \bar{u}(\boldsymbol{x}) \cdot \boldsymbol{n}_{K, \sigma}(\boldsymbol{x}) \mathrm{d} s(\boldsymbol{x})$, and $\boldsymbol{n}_{K, \sigma}(\boldsymbol{x})$ is the outward unit normal vector to $\sigma$ at the point $\boldsymbol{x}$. An intense research activity has recently been devoted to the numerical approximation $\Phi_{K, \sigma}$ of $\bar{\Phi}_{K, \sigma}(\bar{u})$, particularly in the oil engineering framework, where such problems are of utmost importance; we refer for instance to the various schemes presented at the FVCA5 and FVCA6 benchmarks [23,25].

In the case where $\Lambda$ is a heterogeneous isotropic diffusion operator, if the mesh satisfies a standard orthogonality condition, then the continuous flux $\bar{\Phi}_{K, \sigma}(\bar{u})$ through the interface $\sigma$ between the control volumes $K$ and $L$ may be consistently approximated by the two points approximation $\Phi_{K, \sigma}=\tau_{K, L}\left(u_{K}-u_{L}\right)$, where $u_{K}$ and $u_{L}$ are the approximate values of $u$ at points $\boldsymbol{x}_{K}$ and $\boldsymbol{x}_{L}$ and where $\tau_{K, L}$ depends on the geometric properties of the mesh and on $\Lambda$ via the harmonic mean value of $\Lambda$ between $K$ and $L$. The standard orthogonality is satisfied in the particular case of rectangular meshes (2D or $3 \mathrm{D})$, or acute angle triangular meshes (note that this does not generalise to 3D tetrahedra), Voronoï meshes (2D or 3D). An advantage of the above two point approximation is that it provides monotonicity properties, under the form of a local maximum principle. The mathematical properties of the resulting scheme are extensively studied in [19] for example.

Unfortunately, it is seldom possible to use a two point flux in the general case. Indeed, the natural diffusion operators involved in underground flows are in general heterogeneous, accounting for the geological constitution of the layers by sedimentation processes. Furthermore, the geometry of the grid cells resulting from the design of the above depicted 3D mesh does not satisfy the aforementioned orthogonality condition needed for the consistency of the two point approximated flux with $\nabla \bar{u} \cdot \boldsymbol{n}_{K, \sigma}$.

In order to obtain a consistent approximation in the general case, a first idea is to use more than two points in the approximation of $\Phi_{K, \sigma}(u)$, but in a careful way so as to keep a conservative flux. This has been the object of several studies [19], Chapter 3, [1,12,24].

However these schemes usually fail to meet two properties which are of some interest in practice, namely symmetry and positive-definiteness. Indeed, except in some particular cases, multi point schemes are not symmetric; recall that the symmetry of the matrices allows for important savings in computing time; moreover, except again in some particular cases, there is no evidence that the matrix obtained with these coefficients is positive definite, and that convergence properties can be expected; in fact, there exist particular meshes on which these methods lead to noninvertible linear systems.

In order to obtain a consistent approximation of the diffusion flux, a second idea is to use a gradient-type scheme. The construction of such a scheme is based on a non-conformal approximation of the weak formulation of the problem. In this sense, it relates to nonconforming finite element methods. Defining the set $X_{\mathcal{D}, 0}$ of all families of discrete unknowns, for $u \in X_{\mathcal{D}, 0}$, we denote by $\nabla_{\mathcal{D}} u \in L^{2}(\Omega)^{d}$ a discrete gradient and by 
$\Pi_{\mathcal{D}} u \in L^{2}(\Omega)$ a reconstruction of a space function. Then Problem (1.1) is approximated by the scheme

$$
u \in X_{\mathcal{D}, 0}, \forall v \in X_{\mathcal{D}, 0}, \int_{\Omega} \Lambda(\boldsymbol{x}) \nabla_{\mathcal{D}} u(\boldsymbol{x}) \cdot \nabla_{\mathcal{D}} v(\boldsymbol{x}) \mathrm{d} \boldsymbol{x}=\int_{\Omega} f(\boldsymbol{x}) \Pi_{\mathcal{D}} v(\boldsymbol{x}) \mathrm{d} \boldsymbol{x}
$$

In Section 2, we present the properties which are sufficient for the convergence of the scheme (1.2). We also give examples of gradient schemes, among which the SUSHI scheme, whose discrete gradient is obtained using the Stokes formula and relies on the use of auxiliary unknowns $u_{\sigma}$ located on the interfaces $\sigma$ between control volumes and a stabilisation in order to obtain a coercive scheme. A drawback of such schemes is the difficulty to eliminate the auxiliary unknowns $u_{\sigma}$ algebraically, in order for the diffusion terms to be only expressed with respect to the unknowns $u_{K}$ [5], leading to unacceptable computational costs in the engineering framework. Therefore, the scheme can be modified, defining the auxiliary unknowns $u_{\sigma}$ as linear interpolations of the unknowns $u_{K}$ meeting some properties with respect to the diffusion operator $\Lambda(\boldsymbol{x})$ (see [21]). Then the scheme becomes cell-centred, but the stencil of the corresponding term $B_{i, j, k}$ becomes too wide.

This analysis leads us to the construction of two new 3D schemes: a hybrid vertex scheme and a cell centred scheme, which we present in this paper. Both meet the advantages of multi point schemes (in particular, a stencil with at most 27 points) and the symmetry and convergence properties of the SUSHI scheme.

The first one, detailed in Section 3, follows the idea proposed in [18] of introducing vertex unknowns, which we adapt to general meshes using the discrete gradient introduced in [21]. Note that the same type of idea was also used in [10]. It is applicable to any polyhedral mesh, whether the faces of the control volumes are planar or not. Its main characteristics are the following:

(1) the discrete unknowns are the values $u_{K}$ at the centre of the control volumes and the values $u_{\boldsymbol{s}}$ at the vertices, allowing to express the auxiliary unknowns $u_{\sigma}$ (used in the discrete gradient) as linear interpolations of the unknowns $u_{\boldsymbol{s}}$;

(2) after a local elimination of the cell unknowns, we get a small stencil on the interior vertices which is 27-point on structured hexahedral meshes;

(3) it provides the exact solution if $\Lambda$ is piecewise constant in polygonal sub-domains and $u$ is affine in each of these sub-domains (this property is sought in the multi-point flux approximation schemes given for instance in [3]);

(4) the scheme is always a gradient scheme in the sense developed in Section 2 and thus the scheme is well posed, leads to a symmetric positive definite matrix, whatever the geometry and the diffusion operator, and the approximate solutions converge to the exact solution of the problem as the grid size tends to 0 , for general heterogeneous anisotropic diffusion problems.

Even though this scheme yields a 27-point linear system on the vertex unknowns, it does not lead to a 27-point linear system on the cell unknowns themselves, which is required in some situations. Hence a second scheme, proposed in Section 4, extends to the 3D case the 2D scheme which was presented in [6]. Its implementation presents a few new difficulties, which have to be overcome. As in the $2 \mathrm{D}$ case, the scheme is based on the socalled harmonic averaging points, which allow for a consistent 2-point interpolation on an interface $\sigma$ between two neighbouring control volumes $K$ and $L$, of any function $u$ whose regularity properties are those of a solution of the diffusion problem. This study is recalled in Section 4.1. Such an interpolation is then used to construct a discrete gradient on the subcells which are delimited by a cell centre, a cell vertex and the two (in 2D) or four (in 3D) harmonic average points of the edges (in 2D) or faces (in 3D) corresponding to the vertex. This discrete gradient is the same (on the subcells) as the one which is used in the SUSHI scheme [21], and therefore, the convergence analysis of the resulting scheme is analogous to that of the SUSHI scheme. However, thanks to the use of the harmonic points and the subcells, its stencil is much smaller. The full scheme is presented in Section 4.2. Its main characteristics are the following:

(1) it may be used on a large class of non-regular polyhedral meshes;

(2) it again provides the exact solution if $\Lambda$ is piecewise constant in polygonal sub-domains and $u$ is affine in each of these sub-domains; 
(3) it leads to a 27-point scheme in the case of quadrilateral meshes which are not too distorted (in a sense involving the diffusion matrix $\Lambda$ );

(4) it is shown to be a gradient scheme.

Numerical results are provided in Section 5 , followed by a short conclusion in Section 6 .

\section{Approximate Gradient SChemes}

The approximate gradient schemes are written on the weak form of the diffusion problem. From the set of discrete unknowns, a function and a discrete gradient are reconstructed: they are defined almost everywhere and are expected to be an approximation of the unknown function of the problem and its gradient. In order for the scheme to be consistent and convergent, the discrete gradient and the reconstruction operator must be carefully chosen with respect to one another. We first give the definition and convergence properties of a gradient scheme and then give some examples of existing schemes that can be seen as gradient schemes.

\subsection{Definition and properties}

Definition 2.1 (approximate gradient discretization and gradient scheme). Let $\Omega$ be a bounded open domain of $\mathbb{R}^{d}$, with $d \in \mathbb{N}^{\star}$. An approximate gradient discretization $\mathcal{D}$ is defined by $\mathcal{D}=\left(X_{\mathcal{D}, 0}, h_{\mathcal{D}}, \Pi_{\mathcal{D}}, \nabla_{\mathcal{D}}\right)$, where:

(1) the set of discrete unknowns $X_{\mathcal{D}, 0}$ is a finite dimensional vector space on $\mathbb{R}$;

(2) the space step $h_{\mathcal{D}} \in(0,+\infty)$ is a positive real number;

(3) the mapping $\Pi_{\mathcal{D}}: X_{\mathcal{D}, 0} \rightarrow L^{2}(\Omega)$ is the reconstruction of the approximate function;

(4) the mapping $\nabla_{\mathcal{D}}: X_{\mathcal{D}, 0} \rightarrow L^{2}(\Omega)^{d}$ is the reconstruction of the gradient of the function; it must be chosen such that $\left\|\nabla_{\mathcal{D}} \cdot\right\|_{L^{2}(\Omega)^{d}}$ is a norm on $X_{\mathcal{D}, 0}$.

Then the coercivity of the discretization is measured through the norm $C_{\mathcal{D}}$ of the linear mapping $\Pi_{\mathcal{D}}$, defined by

$$
C_{\mathcal{D}}=\max _{v \in X_{\mathcal{D}, 0} \backslash\{0\}} \frac{\left\|\Pi_{\mathcal{D}} v\right\|_{L^{2}(\Omega)}}{\left\|\nabla_{\mathcal{D}} v\right\|_{L^{2}(\Omega)^{d}}} .
$$

Note that (2.1) yields the following "discrete Poincaré" inequality:

$$
\left\|\Pi_{\mathcal{D}} v\right\|_{L^{2}(\Omega)} \leq C_{\mathcal{D}}\left\|\nabla_{\mathcal{D}} v\right\|_{L^{2}(\Omega)^{d}}, \forall v \in X_{\mathcal{D}, 0}
$$

The strong consistency of the discretization is measured through the interpolation error function $S_{\mathcal{D}}: H_{0}^{1}$ $(\Omega) \rightarrow[0,+\infty)$, defined by

$$
S_{\mathcal{D}}(\varphi)=\min _{v \in X_{\mathcal{D}, 0}}\left(\left\|\Pi_{\mathcal{D}} v-\varphi\right\|_{L^{2}(\Omega)}^{2}+\left\|\nabla_{\mathcal{D}} v-\nabla \varphi\right\|_{L^{2}(\Omega)^{d}}^{2}\right)^{\frac{1}{2}}, \forall \varphi \in H_{0}^{1}(\Omega)
$$

The dual consistency of the discretization is measured through the function $W_{\mathcal{D}}: H_{\text {div }}(\Omega) \rightarrow[0,+\infty)$, defined by

$$
W_{\mathcal{D}}(\boldsymbol{\varphi})=\max _{u \in X_{\mathcal{D}, 0 \backslash\{0\}}} \frac{1}{\left\|\nabla_{\mathcal{D}} u\right\|_{L^{2}(\Omega)^{d}}}\left|\int_{\Omega}\left(\nabla_{\mathcal{D}} u(\boldsymbol{x}) \cdot \boldsymbol{\varphi}(\boldsymbol{x})+\Pi_{\mathcal{D}} u(\boldsymbol{x}) \operatorname{div} \boldsymbol{\varphi}(\boldsymbol{x})\right) \mathrm{d} \boldsymbol{x}\right|, \quad \forall \boldsymbol{\varphi} \in H_{\mathrm{div}}(\Omega) .
$$

If $\mathcal{D}=\left(X_{\mathcal{D}, 0}, h_{\mathcal{D}}, \Pi_{\mathcal{D}}, \nabla_{\mathcal{D}}\right)$ is an approximate gradient discretization, we shall say that (1.2) is a gradient scheme.

The following lemma may be seen as the so-called "Strang second lemma", which was introduced in the framework of non nonconforming finite element approximations [29]. We give its formulation in the present setting (along with its proof, which is quite simple). 
Lemma 2.2 (control of the approximation error). Let $\Omega$ be a bounded open domain of $\mathbb{R}^{d}$, with $d \in \mathbb{N}^{\star}$, let $f \in L^{2}(\Omega)$ and let $\Lambda$ be a measurable function from $\Omega$ to the set $\mathcal{M}_{d}(\mathbb{R})$ of $d \times d$ matrices, such that for a.e. $\boldsymbol{x} \in \Omega, \Lambda(\boldsymbol{x})$ is symmetric, and such that the set of its eigenvalues is included in $[\underline{\lambda}, \bar{\lambda}]$, where $0<\underline{\lambda} \leq \bar{\lambda}$. Let $\bar{u} \in H_{0}^{1}(\Omega)$ be the solution of (1.1) (remark that since $f \in L^{2}(\Omega)$, one has $\Lambda \nabla \bar{u} \in H_{\text {div }}(\Omega)$ ).

Let $\mathcal{D}$ be an approximate gradient discretization in the sense of Definition 2.1. Then there exists one and only one $u_{\mathcal{D}} \in X_{\mathcal{D}, 0}$, solution to the approximate gradient scheme (1.2), which moreover satisfies the following inequalities:

$$
\left\|\nabla \bar{u}-\nabla_{\mathcal{D}} u_{\mathcal{D}}\right\|_{L^{2}(\Omega)^{d}} \leq \frac{1}{\underline{\lambda}}\left(W_{\mathcal{D}}(\Lambda \nabla \bar{u})+(\bar{\lambda}+\underline{\lambda}) S_{\mathcal{D}}(\bar{u})\right),
$$

and

$$
\left\|\bar{u}-\Pi_{\mathcal{D}} u_{\mathcal{D}}\right\|_{L^{2}(\Omega)} \leq \frac{1}{\underline{\lambda}}\left(C_{\mathcal{D}} W_{\mathcal{D}}(\Lambda \nabla \bar{u})+\left(C_{\mathcal{D}} \bar{\lambda}+\underline{\lambda}\right) S_{\mathcal{D}}(\bar{u})\right) .
$$

Proof. Assume that $u \in X_{\mathcal{D}, 0}$ satisfies (1.2); taking $v=u$ in (1.2) and using (2.1), we get

$$
\int_{\Omega} \Lambda(\boldsymbol{x}) \nabla_{\mathcal{D}} u(\boldsymbol{x}) \cdot \nabla_{\mathcal{D}} u(\boldsymbol{x}) \mathrm{d} \boldsymbol{x}=\int_{\Omega} f(\boldsymbol{x}) \Pi_{\mathcal{D}} u(\boldsymbol{x}) \mathrm{d} \boldsymbol{x} \leq\|f\|_{L^{2}(\Omega)} C_{\mathcal{D}}\left\|\nabla_{\mathcal{D}} u\right\|_{L^{2}(\Omega)^{d}}
$$

This leads, thanks to the hypotheses on $\Lambda$, to

$$
\underline{\lambda}\left\|\nabla_{\mathcal{D}} u\right\|_{L^{2}(\Omega)^{d}} \leq\|f\|_{L^{2}(\Omega)} C_{\mathcal{D}}
$$

hence, taking $f=0$ yields $\nabla_{\mathcal{D}} u=0$. Thanks to the hypotheses on the approximate gradient, $\left\|\nabla_{\mathcal{D}} \cdot\right\|_{L^{2}(\Omega)^{d}}$ is a norm on $X_{\mathcal{D}, 0}$, we thus get $u=0$. Remarking that the discrete system (1.2) leads to a square linear system, we therefore obtain the existence and uniqueness of $u \in X_{\mathcal{D}, 0}$ solution to (1.2).

Let us take in (2.3), $\varphi=\Lambda \nabla \bar{u} \in H_{\text {div }}(\Omega)$. We then obtain, for a given $v \in X_{\mathcal{D}, 0}$,

$$
\left|\int_{\Omega}\left(\nabla_{\mathcal{D}} v(\boldsymbol{x}) \cdot \Lambda(\boldsymbol{x}) \nabla \bar{u}(\boldsymbol{x})+\Pi_{\mathcal{D}} v(\boldsymbol{x}) \operatorname{div}(\Lambda(\boldsymbol{x}) \nabla \bar{u})(\boldsymbol{x})\right) \mathrm{d} \boldsymbol{x}\right| \leq\left\|\nabla_{\mathcal{D}} v\right\|_{L^{2}(\Omega)^{d}} W_{\mathcal{D}}(\Lambda \nabla \bar{u}),
$$

which leads, since $\bar{u}$ is the solution to (1.1), to

$$
\left|\int_{\Omega}\left(\nabla_{\mathcal{D}} v(\boldsymbol{x}) \cdot \Lambda(\boldsymbol{x}) \nabla \bar{u}(\boldsymbol{x})-\Pi_{\mathcal{D}} v(\boldsymbol{x}) f(\boldsymbol{x})\right) \mathrm{d} \boldsymbol{x}\right| \leq\left\|\nabla_{\mathcal{D}} v\right\|_{L^{2}(\Omega)^{d}} W_{\mathcal{D}}(\Lambda \nabla \bar{u}) .
$$

Since $u_{\mathcal{D}}$ is the unique solution to $(1.2)$, we get

$$
\left|\int_{\Omega} \Lambda \nabla_{\mathcal{D}} v \cdot\left(\nabla \bar{u}-\nabla_{\mathcal{D}} u_{\mathcal{D}}\right) \mathrm{d} \boldsymbol{x}\right| \leq\left\|\nabla_{\mathcal{D}} v\right\|_{L^{2}(\Omega)^{d}} W_{\mathcal{D}}(\Lambda \nabla \bar{u}) .
$$

Defining

$$
P_{\mathcal{D}} \bar{u}=\arg \min _{v \in X_{\mathcal{D}, 0}}\left(\left\|\Pi_{\mathcal{D}} v-\bar{u}\right\|_{L^{2}(\Omega)}^{2}+\left\|\nabla_{\mathcal{D}} v-\nabla \bar{u}\right\|_{L^{2}(\Omega)^{d}}^{2}\right),
$$

and thanks to (2.2), we get

$$
\begin{aligned}
\left|\int_{\Omega} \Lambda \nabla_{\mathcal{D}} v \cdot\left(\nabla_{\mathcal{D}} P_{\mathcal{D}} \bar{u}-\nabla_{\mathcal{D}} u_{\mathcal{D}}\right) \mathrm{d} \boldsymbol{x}\right| & \leq\left\|\nabla_{\mathcal{D}} v\right\|_{L^{2}(\Omega)^{d}} W_{\mathcal{D}}(\Lambda \nabla \bar{u})+\left|\int_{\Omega} \Lambda \nabla_{\mathcal{D}} v \cdot\left(\nabla_{\mathcal{D}} P_{\mathcal{D}} \bar{u}-\nabla \bar{u}\right) \mathrm{d} \boldsymbol{x}\right| \\
& \leq\left\|\nabla_{\mathcal{D}} v\right\|_{L^{2}(\Omega)^{d}}\left(W_{\mathcal{D}}(\Lambda \nabla \bar{u})+\bar{\lambda}\left\|\nabla_{\mathcal{D}} P_{\mathcal{D}} \bar{u}-\nabla \bar{u}\right\|_{L^{2}(\Omega)^{d}}\right) \\
& \leq\left\|\nabla_{\mathcal{D}} v\right\|_{L^{2}(\Omega)^{d}}\left(W_{\mathcal{D}}(\Lambda \nabla \bar{u})+\bar{\lambda} S_{\mathcal{D}}(\bar{u})\right) .
\end{aligned}
$$


Choosing $v=P_{\mathcal{D}} \bar{u}-u_{\mathcal{D}}$ yields

$$
\underline{\lambda}\left\|\nabla_{\mathcal{D}}\left(P_{\mathcal{D}} \bar{u}-u_{\mathcal{D}}\right)\right\|_{L^{2}(\Omega)^{d}} \leq W_{\mathcal{D}}(\Lambda \nabla \bar{u})+\bar{\lambda} S_{\mathcal{D}}(\bar{u})
$$

Remarking that $\left\|\nabla \bar{u}-\nabla_{\mathcal{D}} P_{\mathcal{D}} \bar{u}\right\|_{L^{2}(\Omega)^{d}} \leq S_{\mathcal{D}}(\bar{u})$, we obtain (2.4). Using (2.1), we get

$$
\underline{\lambda}\left\|\Pi_{\mathcal{D}} P_{\mathcal{D}} \bar{u}-\Pi_{\mathcal{D}} u_{\mathcal{D}}\right\|_{L^{2}(\Omega)} \leq C_{\mathcal{D}}\left(W_{\mathcal{D}}(\Lambda \nabla \bar{u})+\bar{\lambda} S_{\mathcal{D}}(\bar{u})\right),
$$

which yields $(2.5)$, since $\left\|\bar{u}-\Pi_{\mathcal{D}} P_{\mathcal{D}} \bar{u}\right\|_{L^{2}(\Omega)^{d}} \leq S_{\mathcal{D}}(\bar{u})$.

We deduce from (2.4) and (2.5) the following convergence result:

Corollary 2.3 (convergence). Under the assumptions of Lemma 2.2, let $\mathcal{F}$ be a family of gradient discretizations in the sense of Definition 2.1, which satisfies the following assumptions:

$(P 1)$ there exists $C_{P} \in \mathbb{R}$ such that $C_{\mathcal{D}} \leq C_{P}$ for any $\mathcal{D} \in \mathcal{F}$;

$(P 2)$ for all $\varphi \in H_{0}^{1}(\Omega), S_{\mathcal{D}}(\varphi)$ tends to 0 as $h_{\mathcal{D}} \rightarrow 0$;

(P3) for all $\varphi \in H_{\mathrm{div}}(\Omega), W_{\mathcal{D}}(\varphi)$ tends to 0 as $h_{\mathcal{D}} \rightarrow 0$.

For $\mathcal{D} \in \mathcal{F}$, let $u_{\mathcal{D}} \in X_{\mathcal{D}, 0}$ be the solution to the approximate gradient scheme $(1.2)$, then $\Pi_{\mathcal{D}} u_{\mathcal{D}}$ converges to $\bar{u}$ in $L^{2}(\Omega)$ and $\nabla_{\mathcal{D}} u_{\mathcal{D}}$ converges to $\nabla \bar{u}$ in $L^{2}(\Omega)^{d}$ as $h_{\mathcal{D}} \rightarrow 0$.

Remark 2.4 (error estimate). In the case where $\bar{u}$ and $\Lambda$ are sufficiently regular, we get for the SUSHI scheme [21] and for the schemes presented in this paper, that $W_{\mathcal{D}}(\Lambda \nabla \bar{u}) \leq C h_{\mathcal{D}}$ and $S_{\mathcal{D}}(\bar{u}) \leq C h_{\mathcal{D}}$, where $C>0$ only depends on the regularity of the mesh.

The following lemma can be used for the practical verification of the assumptions (P2) and (P3) of Corollary 2.3.

Lemma 2.5. Let $\Omega$ be a bounded open domain of $\mathbb{R}^{d}$, with $d \in \mathbb{N}^{\star}$. Let $\mathcal{F}$ be a family of approximate gradient discretizations in the sense of Definition 2.1 Then, for any dense subspace $\mathcal{R}$ of $H_{0}^{1}(\Omega)$, the two properties:

$$
\lim _{h_{\mathcal{D}} \rightarrow 0} S_{\mathcal{D}}(\varphi)=0, \forall \varphi \in \mathcal{R}
$$

and

$$
\lim _{h_{\mathcal{D}} \rightarrow 0} S_{\mathcal{D}}(u)=0, \forall u \in H_{0}^{1}(\Omega),
$$

are equivalent. Furthermore, if there exists $C_{P}>0$ such that the following uniform discrete Poincaré inequality holds:

$$
C_{\mathcal{D}} \leq C_{P}, \forall \mathcal{D} \in \mathcal{F}
$$

then, for any dense subspace $\mathcal{S}$ of $H_{\mathrm{div}}(\Omega)$, the two properties:

$$
\lim _{h_{\mathcal{D}} \rightarrow 0} W_{\mathcal{D}}(\varphi)=0, \forall \varphi \in \mathcal{S}
$$

and

are equivalent.

$$
\lim _{h_{\mathcal{D}} \rightarrow 0} W_{\mathcal{D}}(\boldsymbol{U})=0, \forall \boldsymbol{U} \in H_{\mathrm{div}}(\Omega)
$$

Proof. Let us prove that if (2.8) is satisfied then $\lim _{h_{\mathcal{D}} \rightarrow 0} S_{\mathcal{D}}(u)=0$ for all $u \in H_{0}^{1}(\Omega)$. Let $\varepsilon>0$ and $\varphi \in \mathcal{R}$, such that $\|u-\varphi\|_{H^{1}(\Omega)} \leq \varepsilon$. Thanks to (2.8), there exists $\eta>0$ such that, for all $\mathcal{D} \in \mathcal{F}$ with $h_{\mathcal{D}} \leq \eta$, then

$$
\min _{v \in X_{\mathcal{D}, 0}}\left(\left\|\Pi_{\mathcal{D}} v-\varphi\right\|_{L^{2}(\Omega)}^{2}+\left\|\nabla_{\mathcal{D}} v-\nabla \varphi\right\|_{L^{2}(\Omega)^{d}}^{2}\right) \leq \varepsilon^{2}
$$


Let us denote by

We then get

$$
P_{\mathcal{D}} \varphi=\arg \min _{v \in X_{\mathcal{D}, 0}}\left(\left\|\Pi_{\mathcal{D}} v-\varphi\right\|_{L^{2}(\Omega)}^{2}+\left\|\nabla_{\mathcal{D}} v-\nabla \varphi\right\|_{L^{2}(\Omega)^{d}}^{2}\right)
$$

$$
\left\|\Pi_{\mathcal{D}} P_{\mathcal{D}} \varphi-u\right\|_{L^{2}(\Omega)}^{2}+\left\|\nabla_{\mathcal{D}} P_{\mathcal{D}} \varphi-\nabla u\right\|_{L^{2}(\Omega)^{d}}^{2} \leq 4 \varepsilon^{2}
$$

This proves that $S_{\mathcal{D}}(u) \leq 2 \varepsilon$, hence showing $\lim _{h_{\mathcal{D}} \rightarrow 0} S_{\mathcal{D}}(u)=0$, which proves the first equivalence.

Let us now prove that, under hypothesis $(2.11)$, for all $\boldsymbol{U} \in H_{\operatorname{div}}(\Omega), \lim _{h_{\mathcal{D}} \rightarrow 0} W_{\mathcal{D}}(\boldsymbol{U})=0$. Let $\varphi \in \mathcal{S}$ such that $\|\boldsymbol{U}-\boldsymbol{\varphi}\|_{H_{\mathrm{div}}(\Omega)} \leq \varepsilon$, which means that $\|\boldsymbol{U}-\boldsymbol{\varphi}\|_{L^{2}(\Omega)^{d}} \leq \varepsilon$ and $\|\operatorname{div} \boldsymbol{U}-\operatorname{div} \boldsymbol{\varphi}\|_{L^{2}(\Omega)} \leq \varepsilon$. Thanks to $(2.11)$, there exists $\eta>0$ such that, for all $\mathcal{D} \in \mathcal{F}$ with $h_{\mathcal{D}} \leq \eta$, then, for all $v \in X_{\mathcal{D}, 0}$,

$$
\left|\int_{\Omega}\left(\nabla_{\mathcal{D}} v(\boldsymbol{x}) \cdot \boldsymbol{\varphi}(\boldsymbol{x})+\Pi_{\mathcal{D}} v(\boldsymbol{x}) \operatorname{div} \boldsymbol{\varphi}(\boldsymbol{x})\right) \mathrm{d} \boldsymbol{x}\right| \leq \varepsilon\left\|\nabla_{\mathcal{D}} v\right\|_{L^{2}(\Omega)^{d}} .
$$

Thanks to the triangle and Cauchy-Schwarz inequalities, we have:

$$
\left|\int_{\Omega}\left(\nabla_{\mathcal{D}} v(\boldsymbol{x}) \cdot \boldsymbol{U}(\boldsymbol{x})+\Pi_{\mathcal{D}} v(\boldsymbol{x}) \operatorname{div} \boldsymbol{U}(\boldsymbol{x})\right) \mathrm{d} \boldsymbol{x}\right| \leq \varepsilon\left\|\nabla_{\mathcal{D}} v\right\|_{L^{2}(\Omega)^{d}}+\varepsilon\left\|\nabla_{\mathcal{D}} v\right\|_{L^{2}(\Omega)^{d}}+\varepsilon\left\|\Pi_{\mathcal{D}} v\right\|_{L^{2}(\Omega)} .
$$

Thanks to Hypothesis (2.10), we get

$$
\left|\int_{\Omega}\left(\nabla_{\mathcal{D}} v(\boldsymbol{x}) \cdot \boldsymbol{U}(\boldsymbol{x})+\Pi_{\mathcal{D}} v(\boldsymbol{x}) \operatorname{div} \boldsymbol{U}(\boldsymbol{x})\right) \mathrm{d} \boldsymbol{x}\right| \leq\left(2+C_{P}\right) \varepsilon\left\|\nabla_{\mathcal{D}} v\right\|_{L^{2}(\Omega)^{d}} .
$$

Therefore $W_{\mathcal{D}}(\boldsymbol{U}) \leq\left(2+C_{P}\right) \varepsilon$, hence showing $(2.12)$.

\subsection{Examples}

Let us first notice that standard conforming finite element discretizations may be seen as approximate gradient discretizations. Indeed, let $V_{h} \subset H_{0}^{1}(\Omega)$ be the usual finite element space spanned by the basis functions $\varphi_{1}, \ldots \varphi_{N}$; the space $X_{\mathcal{D}, 0}$ is then $\mathbb{R}^{N}$ and for $u=\left(u_{1}, \ldots, u_{N}\right) \in X_{\mathcal{D}, 0}, \Pi_{\mathcal{D}} u=\sum_{i=1}^{N} u_{i} \varphi_{i}$, and $\nabla_{\mathcal{D}} u=$ $\sum_{i=1}^{N} u_{i} \nabla \varphi_{i}=\nabla \Pi_{\mathcal{D}} u$. Hence

$$
W_{\mathcal{D}}(\varphi)=0 \text { for all } \varphi \in H_{\mathrm{div}}(\Omega)
$$

Note that in fact, an approximate gradient discretization is conforming if and only if (2.13) holds.

Let us now turn to the case of the non conforming P1 finite element discretization on simplicial meshes. In this case, the basis functions of the finite element space $V_{h}$ are associated with the $N$ internal faces of the mesh, and $V_{h}$ is spanned by the basis functions $\varphi_{1}, \ldots, \varphi_{N}$ which are piecewise affine and continuous at the centre of gravity of the faces. In this case, the space $X_{\mathcal{D}, 0}$ is again $\mathbb{R}^{N}$ and for $u=\left(u_{1}, \ldots, u_{N}\right) \in X_{\mathcal{D}, 0}$, $\Pi_{\mathcal{D}} u=\sum_{i=1}^{N} u_{i} \varphi_{i}$, but $\nabla_{\mathcal{D}} u$ cannot be defined as in the conformal case; it is only piecewise defined as the gradient of $\Pi_{\mathcal{D}} u$. It is possible, under some geometrical conditions on the mesh (see e.g. [17]), to get from classical results that for all $\varphi \in\left(C^{1}\left(\mathbb{R}^{d}\right)\right)^{d}, W_{\mathcal{D}}(\boldsymbol{\varphi}) \leq h_{\mathcal{D}} C_{\boldsymbol{\varphi}}$.

The SUSHI scheme [21] is explicitly defined through the space $X_{\mathcal{D}, 0}$, the reconstruction operator $\Pi_{\mathcal{D}}$ and the discrete gradient $\nabla_{\mathcal{D}}$. The fact that $W_{\mathcal{D}}$ tends to 0 may be proved in a similar way to that of the proof of Lemma 3.1 below. Note that the SUSHI scheme is also part of the mimetic mixed hybrid family [16]; however, in the general form of mimetic schemes, we do not know how to include the stabilisation term (which is needed for the coercivity of the scheme) in the gradient term in order to write the scheme under the form (1.2).

The DDFV scheme, see $[9,15,26]$ for the two dimensional case and $[7,8,11,13,14,27,28]$ for the three dimensional case, may also be seen as a gradient scheme. Consider the case where the domain $\Omega$ is the union of octahedra which are the so-called diamond cells (such a cell is depicted in Fig. 2). Octahedral meshes may be obtained from general hexahedral meshes by introducing an internal point to each hexahedron. We show in 

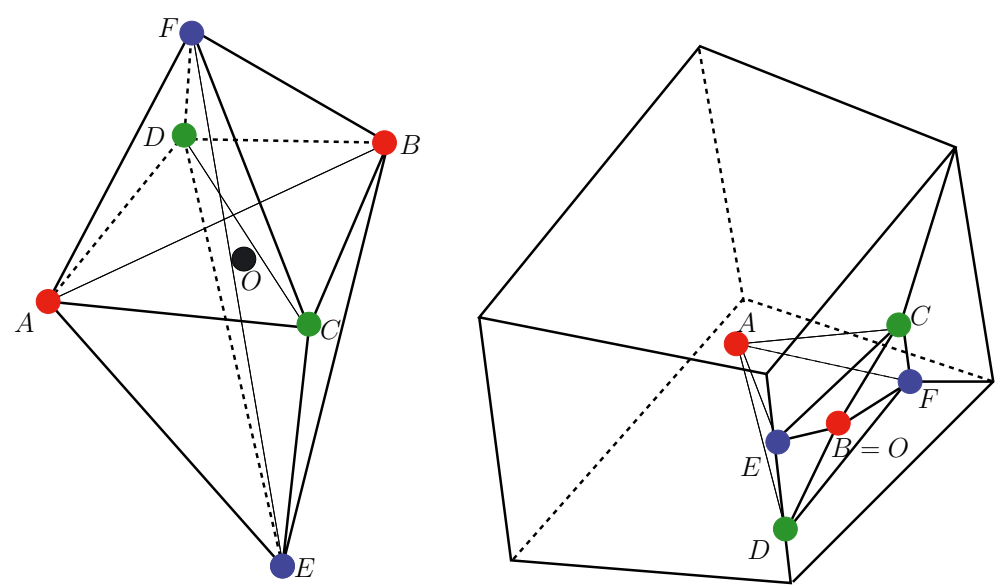

FiguRE 2. Left: a generic octahedral cell for the DDFV scheme - right: an example of construction of an octahedron from a locally refined face of a hexahedron

Figure 2 a locally refined face of hexahedral cell where we depict a octahedron constructed with an internal point of the cell and the barycentre of the four points of a face. (Here and throughout the paper, unless otherwise stated, "barycentre" will refer to a barycentre with equal weights). With such a construction, we can easily take into account boundary conditions and heterogeneous media (each octahedron is homogeneous). The unknown at the centre of the internal faces (point $B$ in right side of Fig. 2), may be easily eliminated. Let us define the space $X_{\mathcal{D}, 0}$ as is $X_{\mathcal{D}, 0}=\left\{\left(u_{\boldsymbol{s}}\right)_{\boldsymbol{s} \in \mathcal{V}}\right\}$, where $\mathcal{V}$ denotes the set of vertices of the octahedral mesh $\mathcal{M}$. Referring to Figure 2, we define a discrete piecewise constant gradient by its value on the octahedron $K$ :

$$
\nabla_{\mathcal{D}} u(\boldsymbol{x})=\frac{1}{\Delta_{K}}\left(\left(u_{B}-u_{A}\right) \overrightarrow{C D} \times \overrightarrow{E F}+\left(u_{D}-u_{C}\right) \overrightarrow{E F} \times \overrightarrow{A B}+\left(u_{F}-u_{E}\right) \overrightarrow{A B} \times \overrightarrow{C D}\right), \quad \forall \boldsymbol{x} \in K
$$

where $\Delta_{K}=\operatorname{Det}(\overrightarrow{A B}, \overrightarrow{C D}, \overrightarrow{E F})$. Let $O$ be a well chosen point in $\bar{K}$, for instance the barycentre of the six vertices $A, B, C, D, E$ and $F$. For the vertex $F$, we denote by $\sigma_{E F}$ the union of the four triangles $O A C, O C B$, $O B D$ and $O D A$, and we denote by $V_{K, F}$ the subset of $K$ of all points which are on the same side of $\sigma_{E F}$ as $F$. We proceed similarly for the five other vertices. The reconstruction operator is then defined for $\boldsymbol{x} \in K$ by:

$$
\Pi_{\mathcal{D}} u(\boldsymbol{x})=\frac{1}{3}\left(u_{A} 1_{V_{K, A}}(\boldsymbol{x})+u_{B} 1_{V_{K, B}}(\boldsymbol{x})+u_{C} 1_{V_{K, C}}(\boldsymbol{x})+u_{D} 1_{V_{K, D}}(\boldsymbol{x})+u_{E} 1_{V_{K, E}}(\boldsymbol{x})+u_{F} 1_{V_{K, F}}(\boldsymbol{x})\right)
$$

where $1_{V}$ is the characteristic function of the set $V$. With these definitions, (1.2) is a DDFV scheme.

\section{A SMALL-STENCIL HYBRID VERTEX SCHEME}

As stated in the introduction, the general idea of the small-stencil hybrid vertex scheme is to introduce a discrete gradient along the same line of thought as that of [20] (this discrete gradient depends on cell and faces unknowns), and to use this discrete gradient to build a discrete bilinear form. The specific point here is to express the face unknowns used in the discrete gradient as linear combinations of the vertex unknowns of the cell. It is then possible to algebraically eliminate the cell unknowns to obtain a linear system involving the vertex unknowns only.

\subsection{Construction of the scheme}

In this section, we provide a definition for all discrete quantities involved in Definition 2.1. We consider generalised polyhedral meshes of $\Omega$. Let $\mathcal{M}$ be the set of control volumes, that are disjoint open subsets of $\Omega$ 
such that $\bigcup_{K \in \mathcal{M}} \bar{K}=\bar{\Omega}$. Let $h_{\mathcal{D}}=\max _{K \in \mathcal{M}} h_{K}$, where $h_{K}$ denotes the diameter of the cell $K$. For all $K$ in $\mathcal{M}, \boldsymbol{x}_{K}$ denotes the so-called "centre" of the control volume $K$ under the assumption that $K$ is star-shaped with respect to $\boldsymbol{x}_{K}$. Let $\mathcal{F}$ denote the set of faces of the mesh; we do not assume that these faces are planar, hence the term "generalised polyhedral". We denote by $\mathcal{V}$ the set of vertices of the mesh. Let $\mathcal{V}_{K}, \mathcal{F}_{K}, \mathcal{V}_{\sigma}$ respectively denote the set of the vertices of $K \in \mathcal{M}$, faces of $K \in \mathcal{M}$, and vertices of $\sigma \in \mathcal{F}$. For any face $\sigma \in \mathcal{F}_{K}$, we have $\mathcal{V}_{\sigma} \subset \mathcal{V}_{K}$

We assume that $\Lambda$ is constant on all $K \in \mathcal{M}$, and we denote by $\Lambda_{K}$ its value in $K$.

We define the set $X_{\mathcal{D}}$ as the set of all real families $u=\left(\left(u_{K}\right)_{K \in \mathcal{M}},\left(u_{\boldsymbol{s}}\right)_{\boldsymbol{s} \in \mathcal{V}}\right)$, and $X_{\mathcal{D}, 0} \subset X_{\mathcal{D}}$ as the set the families $u=\left(\left(u_{K}\right)_{K \in \mathcal{M}},\left(u_{\boldsymbol{s}}\right)_{\boldsymbol{s} \in \mathcal{V})}\right)$ such that $u_{\boldsymbol{s}}=0$ if $\boldsymbol{s}$ is a boundary vertex. Each face $\sigma \in \mathcal{F}$ with vertices $\left\{s \in \mathcal{V}_{\sigma}\right\}$, is decomposed into planar subfaces $\tau$; there are several ways to obtain such a decomposition, possibly using auxiliary interior vertices which are then defined as barycentric combinations of the original vertices $s \in \mathcal{V}_{\sigma}$ with non-negative weights; these auxiliary vertices are not in $\mathcal{V}_{\sigma}$. Once these triangular subfaces are chosen, the centre of gravity $\boldsymbol{x}_{\tau}$ of each subface $\tau$ can then be expressed by the following barycentric combination:

$$
\boldsymbol{x}_{\tau}=\sum_{\boldsymbol{s} \in \mathcal{V}_{\sigma}} \beta_{\tau, \boldsymbol{s}} s \text { with } \sum_{\boldsymbol{s} \in \mathcal{V}_{\sigma}} \beta_{\tau, \boldsymbol{s}}=1
$$

where $\beta_{\tau, s} \geq 0$ for all $s \in \mathcal{V}_{\sigma}$. We then define $\beta_{\tau, s}=0$ for all $s \in \mathcal{V} \backslash \mathcal{V}_{\sigma}$. Next, we reconstruct a value $u_{\tau}$ at the point $\boldsymbol{x}_{\tau}$, by $u_{\tau}=\sum_{\boldsymbol{s} \in \mathcal{V}_{\sigma}} \beta_{\tau, s} u_{\boldsymbol{s}}$.

We denote by $T_{K}$ the set of all subfaces of the faces of $K$ and by $d_{K, \tau}$ the Euclidean distance between $\boldsymbol{x}_{K}$ and $\tau$. The definition of a consistent expression for the approximation of the gradient, see [21], relies on the following consequence of the Stokes formula

$$
\frac{1}{|K|} \sum_{\tau \in T_{K}}|\tau|\left(\boldsymbol{x}_{\tau}-\boldsymbol{x}_{K}\right) \boldsymbol{n}_{K, \tau}^{t}=\mathrm{Id}
$$

which implies

$$
\sum_{\boldsymbol{s} \in \mathcal{V}_{K}} \boldsymbol{b}_{K, \boldsymbol{s}}\left(\boldsymbol{s}-\boldsymbol{x}_{K}\right)^{t}=\mathrm{Id}
$$

where

$$
\boldsymbol{b}_{K, \boldsymbol{s}}=\frac{1}{|K|} \sum_{\tau \in T_{K}} \beta_{\tau, \boldsymbol{s}}|\tau| \boldsymbol{n}_{K, \tau} .
$$

The equality (3.1) suggests the following constant value as an approximation of the gradient on cell $K$.

$$
\nabla_{K} u=\frac{1}{|K|} \sum_{\tau \in T_{K}}|\tau|\left(u_{\tau}-u_{K}\right) \boldsymbol{n}_{K, \tau}=\sum_{\boldsymbol{s} \in \mathcal{V}_{K}}\left(u_{\boldsymbol{s}}-u_{K}\right) \boldsymbol{b}_{K, \boldsymbol{s}}, \forall u \in X_{\mathcal{D}} .
$$

However, it is readily seen that this approximate gradient will not yield a gradient scheme: the coefficient of $u_{K}$ in the above expression is zero; moreover it may be equal to zero on all cells for a non constant set of unknowns $\left(u_{\boldsymbol{s}}\right)_{\boldsymbol{s} \in \mathcal{V}}$ (checker-board modes). In fact, on a regular grid, it resumes to the centred gradient.

Let $\left(M_{K, s}\right)_{s \in \mathcal{V}_{K}}$ be a partition of $K$ such that $\left|M_{K, s}\right|=|K| / N_{K}$, where $N_{K}$ is the number of vertices of $K$ (there is no need to define this partition precisely). We first introduce the following consistency error:

$$
R_{K, s} u=u_{s}-u_{K}-\nabla_{K} u \cdot\left(s-x_{K}\right),
$$

and we define, for a given $\gamma>0$, the constant value $\nabla_{K, s} u$ in $M_{K, s}$ :

$$
\nabla_{K, \boldsymbol{s}} u=\nabla_{K} u+\gamma R_{K, \boldsymbol{s}} u \boldsymbol{b}_{K, \boldsymbol{s}}, \forall u \in X_{\mathcal{D}}
$$

In the numerical implementation, $\gamma$ was set to 5 , after some sensitivity tests.

We now define a piecewise constant gradient by $\nabla_{\mathcal{D}} u(\boldsymbol{x})=\nabla_{K, \boldsymbol{s}} u$ for a.e. $\boldsymbol{x} \in M_{K, \boldsymbol{s}}$. 
Let us then define the reconstruction operator $\Pi_{\mathcal{D}}$. If we wish the scheme to be a finite volume scheme, we choose the piecewise constant reconstruction operator defined by: $\Pi_{\mathcal{D}}^{0} u(\boldsymbol{x})=u_{K}$ for a.e. $\boldsymbol{x} \in K$. In the case where second order convergence is observed at the points $x_{K}$, the piecewise affine reconstruction defined by $\Pi_{\mathcal{D}} u(\boldsymbol{x})=u_{K}+\nabla_{K} u \cdot\left(\boldsymbol{x}-\boldsymbol{x}_{K}\right)$ for a.e. $\boldsymbol{x} \in K$ yields a better approximate solution. We shall only perform the convergence analysis with this latter choice since the analysis for the former choice is simpler.

\subsection{Implementation of the scheme}

We remark that scheme (1.2) may also be written

$$
\text { find } u \in X_{\mathcal{D}, 0}, \forall v \in X_{\mathcal{D}, 0}, \sum_{K \in \mathcal{M}} \sum_{\boldsymbol{s} \in \mathcal{V}_{K}}\left|M_{K, \boldsymbol{s}}\right| \Lambda_{K} \nabla_{K, \boldsymbol{s}} u \cdot \nabla_{K, \boldsymbol{s}} v=\sum_{K \in \mathcal{M}} \int_{K}\left(v_{K}+\nabla_{K} v \cdot\left(\boldsymbol{x}-\boldsymbol{x}_{K}\right)\right) f(\boldsymbol{x}) \mathrm{d} \boldsymbol{x} \text {. }
$$

From Section 3.1, we get that $\nabla_{K, s} u$ can be written under the form

$$
\nabla_{K, \boldsymbol{s}} u=\sum_{\boldsymbol{s}^{\prime} \in \mathcal{V}_{K}}\left(u_{\boldsymbol{s}^{\prime}}-u_{K}\right) \boldsymbol{g}_{K, \boldsymbol{s}}^{\boldsymbol{s}^{\prime}}
$$

with

Defining

$$
\boldsymbol{g}_{K, \boldsymbol{s}}^{\boldsymbol{s}^{\prime}}=\boldsymbol{b}_{K, \boldsymbol{s}^{\prime}}-\gamma \boldsymbol{b}_{K, \boldsymbol{s}}\left(\boldsymbol{s}-\boldsymbol{x}_{K}\right)^{t} \boldsymbol{b}_{K, \boldsymbol{s}^{\prime}}+\gamma \delta_{\boldsymbol{s}, \boldsymbol{s}^{\prime}} \boldsymbol{b}_{K, \boldsymbol{s}}
$$

$$
A_{K}^{s, s^{\prime}}=\sum_{\boldsymbol{s}^{\prime \prime} \in \mathcal{V}_{K}}\left|M_{K, \boldsymbol{s}^{\prime \prime}}\right| \Lambda_{K} \boldsymbol{g}_{K, \boldsymbol{s}^{\prime \prime}}^{\boldsymbol{s}} \cdot \boldsymbol{g}_{K, \boldsymbol{s}^{\prime \prime}}^{\boldsymbol{s}^{\prime}}
$$

we get that

$$
\sum_{K \in \mathcal{M}} \sum_{\boldsymbol{s} \in \mathcal{V}_{K}}\left|M_{K, \boldsymbol{s}}\right| \Lambda_{K} \nabla_{K, \boldsymbol{s}} u \cdot \nabla_{K, \boldsymbol{s}} v=\sum_{K \in \mathcal{M}} \sum_{\boldsymbol{s} \in \mathcal{V}_{K}} \sum_{\boldsymbol{s}^{\prime} \in \mathcal{V}_{K}} A_{K}^{\boldsymbol{s}, \boldsymbol{s}^{\prime}}\left(u_{\boldsymbol{s}}-u_{K}\right)\left(v_{\boldsymbol{s}^{\prime}}-v_{k}\right)
$$

Considering the test function $v \in X_{\mathcal{D}, 0}$ such that the only non zero component of $v$ is $v_{K}=1$, the above expression leads to a linear equation which only depends on $u_{K}$ and $u_{s}$ for $s \in \mathcal{V}_{K}$, hence allowing a local elimination of $u_{K}$. Then a linear system on all $u_{\boldsymbol{s}}, \boldsymbol{s} \in \mathcal{V}$ is obtained by the elimination of all the cell values $u_{K}$, resulting in a 27-point stencil on a hexahedral mesh.

\subsection{Mathematical properties}

Lemma 3.1 (gradient scheme properties of the vertex scheme). Let $\mathcal{D}=\left(X_{\mathcal{D}, 0}, h_{\mathcal{D}}, \Pi_{\mathcal{D}}, \nabla_{\mathcal{D}}\right)$ be a discretization in the sense of Definition 2.1 with $X_{\mathcal{D}, 0}, h_{\mathcal{D}}, \Pi_{\mathcal{D}}, \nabla_{\mathcal{D}}$ defined in Section 3.1, then, for a fixed $\gamma$, the scheme (1.2) is a gradient scheme in the sense of Definition 2.1, and therefore there exists a unique solution to (1.2). Furthermore, if $\mathcal{F}$ is a family of such discretizations for which there exists $\theta>0$ such that, for all $\mathcal{D} \in \mathcal{F}$ :

$$
\left\{\begin{array}{l}
\theta \leq \frac{d_{K, \tau}}{d_{L, \tau}} \leq 1 / \theta \text { for all neighbouring cells } K \text { and } L \text { and for all } \tau \in T_{K} \cap T_{L}, \\
d_{K, \tau}>\theta h_{K} \text { for all grid cells } K \in \mathcal{M} \text { and } \tau \in T_{K}, \\
N_{K} \leq \frac{1}{\theta}, \text { for all grid cells } K \in \mathcal{M}, \\
h_{K}\left|\boldsymbol{b}_{K, \boldsymbol{s}}\right| \geq \theta, \text { for all grid cells } K \in \mathcal{M} \text { and } \boldsymbol{s} \in \mathcal{V}_{K},
\end{array}\right.
$$

the property (P1) of Corollary 2.3 is satisfied. Moreover,

$$
S_{\mathcal{D}}(\varphi) \leq C_{\varphi} h_{\mathcal{D}}, \forall \varphi \in C_{0}^{2}(\bar{\Omega}), \forall \mathcal{D} \in \mathcal{F}
$$


where $C_{0}^{2}(\bar{\Omega})$ is the set of $C^{2}$ functions which vanish on $\partial \Omega$, and

$$
W_{\mathcal{D}}(\boldsymbol{\varphi}) \leq C_{\varphi} h_{\mathcal{D}}, \forall \varphi \in\left(C^{1}\left(\overline{\mathbb{R}}^{d}\right)\right)^{d}, \forall \mathcal{D} \in \mathcal{F}
$$

Therefore properties (P2) and (P3) of Corollary 2.3 are satisfied: the scheme (1.2) is convergent and an error estimate holds in the case of regular solutions.

Proof. We first need to prove that there exists $C_{P}$ not depending on the discretization $\mathcal{D}$, such that

$$
\left\|\Pi_{\mathcal{D}} v\right\|_{L^{2}(\Omega)} \leq C_{P}\left\|\nabla_{\mathcal{D}} v\right\|_{L^{2}(\Omega)^{d}}, \forall v \in X_{\mathcal{D}, 0}
$$

which shows that $C_{\mathcal{D}}$ remains bounded by $C_{P}$.

From the discrete Sobolev embeddings proved in [21], Lemma 5.3, we have the following discrete Poincaré inequality:

$$
\left\|\Pi_{\mathcal{D}}^{0} v\right\|_{L^{2}(\Omega)} \leq C_{1}\|v\|_{1, \mathcal{D}}, \forall v \in X_{\mathcal{D}, 0}
$$

where $\Pi_{\mathcal{D}}^{0} v$ is the piecewise constant reconstruction of $v$ defined by: $\Pi_{\mathcal{D}}^{0} v(x)=v_{K}$ for $\boldsymbol{x} \in K, C_{1} \geq 0$ depends on $\Omega$ and $\theta$ only and $\|\cdot\|_{1, \mathcal{D}}$ is the discrete $H^{1}$ norm defined by:

$$
\|v\|_{1, \mathcal{D}}^{2}=\sum_{K \in \mathcal{M}} \sum_{\tau \in T_{K}} \frac{|\tau|}{d_{K, \tau}}\left(v_{\tau}-v_{K}\right)^{2}
$$

Let us then show that $\|v\|_{1, \mathcal{D}} \leq C_{2}\left\|\nabla_{\mathcal{D}} v\right\|_{L^{2}(\Omega)^{d}}$ for any $v \in X_{\mathcal{D}, 0}$, where $C_{2}$ only depends on $\Omega, \theta$ and $\gamma$. Thanks to the fact that $\left|M_{K, s}\right|=\frac{|K|}{N_{K}}$, we have

$$
\int_{\Omega}\left|\nabla_{\mathcal{D}} v\right|^{2} \mathrm{~d} \boldsymbol{x}=\sum_{K \in \mathcal{M}} \sum_{\boldsymbol{s} \in \mathcal{V}_{K}} \frac{|K|}{N_{K}}\left|\nabla_{K} v+\gamma R_{K, \boldsymbol{s}} v \boldsymbol{b}_{K, \boldsymbol{s}}\right|^{2}
$$

But, thanks to (3.1), we get:

$$
\sum_{\boldsymbol{s} \in \mathcal{V}_{K}} R_{K, \boldsymbol{s}} v \boldsymbol{b}_{K, \boldsymbol{s}}=\sum_{\boldsymbol{s} \in \mathcal{V}_{K}}\left(v_{\boldsymbol{s}}-v_{K}\right) \boldsymbol{b}_{K, \boldsymbol{s}}-\sum_{\boldsymbol{s} \in \mathcal{V}_{K}}\left(\boldsymbol{s}-\boldsymbol{x}_{K}\right) \boldsymbol{b}_{K, \boldsymbol{s}}^{t} \nabla_{K} v=\nabla_{K} v-\nabla_{K} v=0
$$

and therefore:

$$
\int_{\Omega}\left|\nabla_{\mathcal{D}} v\right|^{2} \mathrm{~d} \boldsymbol{x}=\sum_{K \in \mathcal{M}}\left(|K|\left|\nabla_{K} v\right|^{2}+\sum_{\boldsymbol{s} \in \mathcal{V}_{K}} \frac{|K|}{N_{K}} \gamma^{2}\left(R_{K, \boldsymbol{s}} v\right)^{2}\left|\boldsymbol{b}_{K, \boldsymbol{s}}\right|^{2}\right) .
$$

Since for all $a, b \in \mathbb{R}$, and $\mu>-1$ we have $\left(\frac{a}{\sqrt{1+\mu}}-b \sqrt{1+\mu}\right)^{2} \geq 0$, we obtain $(a-b)^{2} \geq \frac{\mu}{1+\mu} a^{2}-\mu b^{2}$, and therefore,

$$
\left(R_{K, \boldsymbol{s}} v\right)^{2} \geq \frac{\mu_{K, \boldsymbol{s}}}{1+\mu_{K, \boldsymbol{s}}}\left(v_{\boldsymbol{s}}-v_{K}\right)^{2}-\mu_{K, \boldsymbol{s}}\left|\nabla_{K} v\right|^{2}\left|\boldsymbol{s}-x_{K}\right|^{2}
$$

for any $\mu_{K, s}>0$. Defining $\mu_{K, s}>0$ by

$$
1-\mu_{K, \boldsymbol{s}} \gamma^{2}\left|\boldsymbol{s}-x_{K}\right|^{2}\left|\boldsymbol{b}_{K, \boldsymbol{s}}\right|^{2}=0
$$

we obtain

$$
\int_{\Omega}\left|\nabla_{\mathcal{D}} v(\boldsymbol{x})\right|^{2} \mathrm{~d} \boldsymbol{x} \geq \gamma^{2} \frac{1}{1+\max _{\substack{K \in \mathcal{M} \\ \boldsymbol{s} \in \mathcal{V}_{K}}} \mu_{K, \boldsymbol{s}}} \sum_{K \in \mathcal{M}} \sum_{\boldsymbol{s} \in \mathcal{V}_{K}} \frac{|K|}{N_{K}} \frac{\left(v_{\boldsymbol{s}}-v_{K}\right)^{2}}{\left|\boldsymbol{s}-x_{K}\right|^{2}}
$$


and therefore, thanks to the regularity assumption (3.5),

$$
\int_{\Omega}\left|\nabla_{\mathcal{D}} v(\boldsymbol{x})\right|^{2} \mathrm{~d} \boldsymbol{x} \geq \gamma^{2} \frac{1}{1+C_{1}} \sum_{K \in \mathcal{M}} \sum_{\boldsymbol{s} \in \mathcal{V}_{K}} \frac{|K|}{N_{K}} \frac{\left(v_{\boldsymbol{s}}-v_{K}\right)^{2}}{\left|\boldsymbol{s}-x_{K}\right|^{2}},
$$

where $C_{1} \in \mathbb{R}_{+}$depends only on $\theta$ and $\gamma$. Now, since we have

$$
\left(v_{\tau}-v_{K}\right)^{2}=\left(\sum_{\boldsymbol{s} \in \mathcal{V}_{K}} \beta_{\tau, \boldsymbol{s}}\left(v_{\boldsymbol{s}}-v_{K}\right)\right)^{2} \leq\left(\sum_{\boldsymbol{s} \in \mathcal{V}_{K}} \beta_{\tau, \boldsymbol{s}}\right)\left(\sum_{\boldsymbol{s} \in \mathcal{V}_{K}} \beta_{\tau, \boldsymbol{s}}\left(v_{\boldsymbol{s}}-v_{K}\right)^{2}\right)=\sum_{\boldsymbol{s} \in \mathcal{V}_{K}} \beta_{\tau, \boldsymbol{s}}\left(v_{\boldsymbol{s}}-v_{K}\right)^{2},
$$

we get

$$
\|v\|_{1, \mathcal{D}}^{2}=\sum_{K \in \mathcal{M}} \sum_{\tau \in T_{K}} \frac{|\tau|}{d\left(x_{K}, \tau\right)}\left(v_{\tau}-v_{K}\right)^{2} \leq \sum_{K \in \mathcal{M}} \sum_{\tau \in T_{K}} \frac{|\tau|}{d\left(x_{K}, \tau\right)} \sum_{s \in \mathcal{V}_{K}} \beta_{\tau, \boldsymbol{s}}\left(v_{\boldsymbol{s}}-v_{K}\right)^{2},
$$

and therefore

$$
\|v\|_{1, \mathcal{D}}^{2} \leq \sum_{K \in \mathcal{M}} \sum_{\boldsymbol{s} \in \mathcal{V}_{K}} C_{K, \boldsymbol{s}}\left(v_{\boldsymbol{s}}-v_{K}\right)^{2} \text { with } C_{K, \boldsymbol{s}}=\sum_{\tau \in T_{K}} \beta_{\tau, \boldsymbol{s}} \frac{|\tau|}{d_{K, \tau}}
$$

Again thanks to the regularity assumption (3.5), we have $C_{K, s} \leq C_{2} \frac{|K|}{N_{K}\left|s-x_{K}\right|^{2}}$ where $C_{2} \in \mathbb{R}_{+}$only depends on $\theta$. Together with (3.10), this yields that there exists $C_{3} \in \mathbb{R}_{+}$only depending on $\Omega, \theta$ and $\gamma$ such that

$$
\|v\|_{1, \mathcal{D}} \leq C_{3}\left\|\nabla_{\mathcal{D}} v\right\|_{L^{2}(\Omega)^{d}}
$$

and therefore, thanks to (3.8):

Thanks to (3.9), we get that

$$
\left\|\Pi_{\mathcal{D}}^{0} v\right\|_{L^{2}(\Omega)} \leq C_{1} C_{2}\left\|\nabla_{\mathcal{D}} v\right\|_{L^{2}(\Omega)^{d}} .
$$

$$
\nabla_{K} v=\frac{1}{|K|} \int_{K} \nabla_{\mathcal{D}} v(\boldsymbol{x}) \mathrm{d} \boldsymbol{x}
$$

and therefore, by the triangle inequality:

$$
\left\|\Pi_{\mathcal{D}} v\right\|_{L^{2}(\Omega)} \leq\left\|\Pi_{\mathcal{D}}^{0} v\right\|_{L^{2}(\Omega)}+h_{\mathcal{D}}\left\|\nabla_{\mathcal{D}} v\right\|_{L^{2}(\Omega)^{d}} .
$$

From the two above inequalities, we conclude the proof of (P1).

Property (P2) is a consequence of the Stokes formula, the proof is similar to that of [21], Lemma 4.3. Let us now prove (P3). Let $\varphi \in\left(C^{1}\left(\mathbb{R}^{d}\right)\right)^{d}$ be given, and let $v \in X_{\mathcal{D}, 0}$. Let us compute

$$
T_{1}=\int_{\Omega}\left(\nabla_{\mathcal{D}} v(\boldsymbol{x}) \cdot \boldsymbol{\varphi}(\boldsymbol{x})+\Pi_{\mathcal{D}} v(\boldsymbol{x}) \operatorname{div} \boldsymbol{\varphi}(\boldsymbol{x})\right) \mathrm{d} \boldsymbol{x} .
$$

Denoting by $\bar{\varphi}_{K}$ the average value of $\varphi$ in $K, \bar{\varphi}_{\tau}$ the average value of $\varphi$ on $\tau$ and $\bar{\varphi}_{K, s}$ the average value of $\varphi$ in $M_{K, \boldsymbol{s}}$, we get $T_{1}=T_{2}+T_{3}+T_{4}$ with

$$
\begin{aligned}
T_{2}=\sum_{K \in \mathcal{M}} \sum_{\boldsymbol{s} \in \mathcal{V}_{K}}\left|M_{K, \boldsymbol{s}}\right| \nabla_{K, \boldsymbol{s}} v \cdot \overline{\boldsymbol{\varphi}}_{K, \boldsymbol{s}}, T_{3}=\sum_{K \in \mathcal{M}} v_{K} \sum_{\tau \in T_{K}}|\tau| \boldsymbol{n}_{K, \tau} \cdot \overline{\boldsymbol{\varphi}}_{\tau} \\
\quad \text { and } T_{4}=\sum_{K \in \mathcal{M}} \int_{K} \nabla_{K} v \cdot\left(\boldsymbol{x}-\boldsymbol{x}_{K}\right) \operatorname{div} \boldsymbol{\varphi}(\boldsymbol{x}) \mathrm{d} \boldsymbol{x} .
\end{aligned}
$$

We have $T_{2}=T_{21}+T_{22}$ with

$$
T_{21}=\sum_{K \in \mathcal{M}} \sum_{\boldsymbol{s} \in \mathcal{V}_{K}} \frac{|K|}{N_{K}} \nabla_{K} v \cdot \overline{\boldsymbol{\varphi}}_{K, \boldsymbol{s}}, \quad \text { and } \quad T_{22}=\sum_{K \in \mathcal{M}} \sum_{\boldsymbol{s} \in \mathcal{V}_{K}} \frac{|K|}{N_{K}} \gamma R_{K, \boldsymbol{s}} v \boldsymbol{b}_{K, \boldsymbol{s}} \cdot \overline{\boldsymbol{\varphi}}_{K, \boldsymbol{s}} .
$$


We get

$$
T_{21}=\sum_{K \in \mathcal{M}}|K| \nabla_{K} v \cdot \overline{\boldsymbol{\varphi}}_{K}=\sum_{K \in \mathcal{M}} \sum_{\tau \in T_{K}}|\tau|\left(v_{\tau}-v_{K}\right) \boldsymbol{n}_{K, \tau} \cdot \overline{\boldsymbol{\varphi}}_{K} .
$$

Remarking that if $\tau$ is on the boundary of $\Omega$ then $v_{\tau}=0$ and that otherwise $v_{\tau}$ appears in two opposite terms from the two neighbouring cells sharing $\tau$, we have

$$
T_{3}=\sum_{K \in \mathcal{M}} \sum_{\tau \in T_{K}}\left(v_{K}-v_{\tau}\right)|\tau| \boldsymbol{n}_{K, \tau} \cdot \overline{\boldsymbol{\varphi}}_{\tau}
$$

we get

$$
T_{21}+T_{3}=\sum_{K \in \mathcal{M}} \sum_{\tau \in T_{K}}\left(v_{K}-v_{\tau}\right)|\tau| \boldsymbol{n}_{K, \tau} \cdot\left(\overline{\boldsymbol{\varphi}}_{\tau}-\overline{\boldsymbol{\varphi}}_{K}\right) .
$$

Hence, thanks to the Cauchy-Schwarz inequality

$$
\left|T_{21}+T_{3}\right| \leq \sqrt{|\Omega|}\|v\|_{1, \mathcal{D}} h_{\mathcal{D}} C_{\varphi}
$$

We then use (3.9), and we get

$$
T_{22}=\sum_{K \in \mathcal{M}} \sum_{\boldsymbol{s} \in \mathcal{V}_{K}} \frac{|K|}{N_{K}} \gamma R_{K, \boldsymbol{s}} v \boldsymbol{b}_{K, \boldsymbol{s}} \cdot\left(\overline{\boldsymbol{\varphi}}_{K, \boldsymbol{s}}-\overline{\boldsymbol{\varphi}}_{K}\right) .
$$

This leads to $\left|T_{22}\right| \leq\left|T_{221}\right|+\left|T_{222}\right|$, with

$$
T_{221}=\sum_{K \in \mathcal{M}} \sum_{\boldsymbol{s} \in \mathcal{V}_{K}} \frac{|K|}{N_{K}} \gamma\left(v_{\boldsymbol{s}}-v_{K}\right) \boldsymbol{b}_{K, \boldsymbol{s}} \cdot\left(\overline{\boldsymbol{\varphi}}_{K, \boldsymbol{s}}-\overline{\boldsymbol{\varphi}}_{K}\right)
$$

and

$$
T_{222}=\sum_{K \in \mathcal{M}} \sum_{\boldsymbol{s} \in \mathcal{V}_{K}} \frac{|K|}{N_{K}} \gamma \nabla_{K} u \cdot\left(\boldsymbol{s}-\boldsymbol{x}_{K}\right) \boldsymbol{b}_{K, \boldsymbol{s}} \cdot\left(\overline{\boldsymbol{\varphi}}_{K, \boldsymbol{s}}-\overline{\boldsymbol{\varphi}}_{K}\right) .
$$

Using the Cauchy-Schwarz inequality and (3.10), we conclude that

$$
\left|T_{22}\right| \leq h_{\mathcal{D}} C_{\varphi}\left\|\nabla_{\mathcal{D}} v\right\|_{L^{2}(\Omega)^{d}}
$$

Turning now to $T_{4}$ and using (3.11), we may write:

$$
T_{4} \leq h_{\mathcal{D}}\left\|\nabla_{\mathcal{D}} v\right\|_{L^{2}(\Omega)^{d}}\|\operatorname{div} \varphi\|_{L^{2}(\Omega)}
$$

From (3.12)-(3.14), we get (3.7), which concludes the proof.

Let us now verify the following consistency property, that is useful to assess the precision of the scheme on coarse meshes often used in the industrial setting: if the continuous problem admits an exact piecewise affine solution, then its interpolate is the solution to the scheme. This property is automatically satisfied when using a conforming approximation such as the P1 finite element scheme, but it must be checked for nonconforming approximations.

Lemma 3.2 (exact solution on piecewise affine functions). Let $\bar{u}=\left(\left(\bar{u}_{K}\right)_{K \in \mathcal{M}},\left(\bar{u}_{\boldsymbol{s}}\right)_{\boldsymbol{s} \in \mathcal{V}}\right) \in X_{\mathcal{D}}$ such that for all $K \in \mathcal{M}$, there exists $\boldsymbol{G}_{K} \in \mathbb{R}^{d}$ with

$$
\bar{u}_{\boldsymbol{s}}-\bar{u}_{K}=\left(\boldsymbol{s}-\boldsymbol{x}_{K}\right)^{t} \cdot \boldsymbol{G}_{K}, \forall K \in \mathcal{M}, \forall \boldsymbol{s} \in \mathcal{V}_{K},
$$


and, such that for any $\tau \in T_{K} \cap T_{L}$ included in the interface between two neighbouring blocks $K$ and $L$,

$$
\Lambda_{K} \boldsymbol{G}_{K} \cdot \boldsymbol{n}_{K, \tau}+\Lambda_{L} \boldsymbol{G}_{L} \cdot \boldsymbol{n}_{L, \tau}=0
$$

Then $\bar{u}$ is the unique solution of the discrete diffusion problem with non homogeneous Dirichlet boundary conditions (existence and uniqueness follow from Lem. 3.1):

$$
u \in X_{\mathcal{D}, 0}+\bar{u}, \forall v \in X_{\mathcal{D}, 0}, \int_{\Omega} \Lambda(\boldsymbol{x}) \nabla_{\mathcal{D}} u(\boldsymbol{x}) \cdot \nabla_{\mathcal{D}} v(\boldsymbol{x}) \mathrm{d} \boldsymbol{x}=0 .
$$

Proof. From the definition (3.4) of $\boldsymbol{g}_{K, \boldsymbol{s}}^{\boldsymbol{s}^{\prime}}$, we have:

$$
\sum_{\boldsymbol{s} \in \mathcal{V}_{K}} \boldsymbol{g}_{K, \boldsymbol{s}}^{\boldsymbol{s}^{\prime}}=N_{K} \boldsymbol{b}_{K, \boldsymbol{s}^{\prime}}-\gamma \boldsymbol{b}_{K, \boldsymbol{s}^{\prime}}+\gamma \boldsymbol{b}_{K, \boldsymbol{s}^{\prime}}=N_{K} \boldsymbol{b}_{K, \boldsymbol{s}^{\prime}}
$$

We also have, thanks to (3.1) and (3.15),

$$
\begin{aligned}
\sum_{\boldsymbol{s}^{\prime} \in \mathcal{V}_{K}}\left(\bar{u}_{\boldsymbol{s}^{\prime}}-\bar{u}_{K}\right) \boldsymbol{g}_{K, \boldsymbol{s}}^{\boldsymbol{s}^{\prime}}= & \sum_{\boldsymbol{s}^{\prime} \in \mathcal{V}_{K}} \boldsymbol{b}_{K, \boldsymbol{s}^{\prime}}\left(\boldsymbol{s}^{\prime}-\boldsymbol{x}_{K}\right)^{t} \boldsymbol{G}_{K} \\
& -\gamma \sum_{\boldsymbol{s}^{\prime} \in \mathcal{V}_{K}} \boldsymbol{b}_{K, \boldsymbol{s}}\left(\boldsymbol{s}-\boldsymbol{x}_{K}\right)^{t} \boldsymbol{b}_{K, \boldsymbol{s}^{\prime}}\left(\boldsymbol{s}^{\prime}-\boldsymbol{x}_{K}\right)^{t} \boldsymbol{G}_{K}+\gamma \boldsymbol{b}_{K, \boldsymbol{s}}\left(\boldsymbol{s}-\boldsymbol{x}_{K}\right)^{t} \boldsymbol{G}_{K}=\boldsymbol{G}_{K} .
\end{aligned}
$$

Let us write the discrete operator associated to an interior vertex $s$ :

$$
E_{\boldsymbol{s}}=\sum_{K \in \mathcal{M}} \sum_{\boldsymbol{s}^{\prime} \in \mathcal{V}_{K}} A_{K}^{\boldsymbol{s}, \boldsymbol{s}^{\prime}}\left(\bar{u}_{\boldsymbol{s}^{\prime}}-\bar{u}_{K}\right)=\sum_{K \in \mathcal{M}} \sum_{\boldsymbol{s}^{\prime} \in \mathcal{V}_{K}} \sum_{\boldsymbol{s}^{\prime \prime} \in \mathcal{V}_{K}}\left|M_{K, \boldsymbol{s}^{\prime \prime}}\right| \Lambda_{K} \boldsymbol{g}_{K, \boldsymbol{s}^{\prime \prime}}^{\boldsymbol{s}} \cdot \boldsymbol{g}_{K, \boldsymbol{s}^{\prime \prime}}^{\boldsymbol{s}^{\prime}}\left(\bar{u}_{\boldsymbol{s}^{\prime}}-\bar{u}_{K}\right) .
$$

We then get, using (3.19), that

$$
E_{\boldsymbol{s}}=\sum_{K \in \mathcal{M}} \sum_{\boldsymbol{s}^{\prime \prime} \in \mathcal{V}_{K}} \frac{|K|}{N_{K}} \Lambda_{K} \boldsymbol{g}_{K, \boldsymbol{s}^{\prime \prime}}^{\boldsymbol{s}} \cdot \boldsymbol{G}_{K}
$$

and then, using (3.18),

$$
E_{\boldsymbol{s}}=\sum_{K \in \mathcal{M}} \frac{|K|}{N_{K}} \Lambda_{K} N_{K} \boldsymbol{b}_{K, \boldsymbol{s}} \cdot \boldsymbol{G}_{K}
$$

Now we get, setting $\boldsymbol{n}_{K, \tau}=0$ if $\tau \notin T_{K}$, defining $T=\bigcup_{K \in \mathcal{M}} T_{K}$ and using (3.2) and (3.16),

$$
E_{\boldsymbol{s}}=\sum_{K \in \mathcal{M}} \Lambda_{K} \sum_{\tau \in T_{K}} \beta_{\tau, \boldsymbol{s}}|\tau| \boldsymbol{n}_{K, \tau} \cdot \boldsymbol{G}_{K}=\sum_{\tau \in T} \beta_{\tau, \boldsymbol{s}}|\tau| \sum_{K \in \mathcal{M}} \Lambda_{K} \boldsymbol{n}_{K, \tau} \cdot \boldsymbol{G}_{K}=0 .
$$

Hence the discrete operator applied to this particular solution and associated to an internal vertex is indeed equal to 0 .

Let us now check that the discrete operator applied to this particular solution and associated to a control volume is also equal to 0 .

$$
E_{K}=-\sum_{\boldsymbol{s} \in \mathcal{V}_{K}} \sum_{\boldsymbol{s}^{\prime} \in \mathcal{V}_{K}} A_{K}^{\boldsymbol{s}, \boldsymbol{s}^{\prime}}\left(\bar{u}_{\boldsymbol{s}^{\prime}}-\bar{u}_{K}\right)=-\sum_{\boldsymbol{s} \in \mathcal{V}_{K}} \sum_{\boldsymbol{s}^{\prime} \in \mathcal{V}_{K}} \sum_{\boldsymbol{s}^{\prime \prime} \in \mathcal{V}_{K}}\left|M_{K, \boldsymbol{s}^{\prime \prime}}\right| \Lambda_{K} \boldsymbol{g}_{K, \boldsymbol{s}^{\prime \prime}}^{\boldsymbol{s}} \cdot \boldsymbol{g}_{K, \boldsymbol{s}^{\prime \prime}}^{\boldsymbol{s}^{\prime}}\left(\bar{u}_{\boldsymbol{s}^{\prime}}-\bar{u}_{K}\right),
$$

then, using (3.19),

$$
E_{K}=-\sum_{\boldsymbol{s} \in \mathcal{V}_{K}} \sum_{\boldsymbol{s}^{\prime \prime} \in \mathcal{V}_{K}} \frac{|K|}{N_{K}} \Lambda_{K} \boldsymbol{g}_{K, \boldsymbol{s}^{\prime \prime}}^{\boldsymbol{s}} \cdot \boldsymbol{G}_{K}
$$


which gives, using (3.18),

$$
E_{K}=-\sum_{\boldsymbol{s} \in \mathcal{V}_{K}} \frac{|K|}{N_{K}} \Lambda_{K} N_{K} \boldsymbol{b}_{K, \boldsymbol{s}} \cdot \boldsymbol{G}_{K}
$$

and then, thanks to $(3.2)$,

$$
E_{K}=-|K| \Lambda_{K} \boldsymbol{G}_{K} \cdot \sum_{\boldsymbol{s} \in \mathcal{V}_{K}} \boldsymbol{b}_{K, \boldsymbol{s}}=-\Lambda_{K} \boldsymbol{G}_{K} \cdot \sum_{\tau \in T_{K}} \sum_{\boldsymbol{s} \in \mathcal{V}_{K}} \beta_{\tau, \boldsymbol{s}}|\tau| \boldsymbol{n}_{K, \tau}
$$

and finally

$$
E_{K}=-\Lambda_{K} \boldsymbol{G}_{K} \cdot \sum_{\tau \in T_{K}}|\tau| \boldsymbol{n}_{K, \tau}=0
$$

Remark 3.3. In the above proof, we showed in fact that $W_{\mathcal{D}}(\Lambda \nabla \widehat{u})=0$, where $\widehat{u} \in H^{1}(\Omega)$ is the piecewise affine solution of the non-homogeneous continuous problem. Furthermore, naturally extending the definition of $S_{\mathcal{D}}$ to $H^{1}(\Omega)$, we also have $S_{\mathcal{D}}(\widehat{u})=0$.

\section{A SMALL-STENCIL CELL-CENTRED SCHEME}

Because of the heterogeneity of $\Lambda$ and the possible coupling with a transport equation, it is often not advisable to apply the above hybrid vertex scheme to the dual cells whose vertices are the cell centres, which would seem to be the simplest approach. Therefore our aim is to again define a scheme by (1.2) and suitable discrete quantities (discrete space, discrete reconstruction, interpolation and gradient), under the constraint that the scheme should be cell centred after the elimination of auxiliary unknowns.

Again, in order for the stencil to remain small (and in particular 27-points on hexahedral structured meshes), the discrete gradient on one given cell should only depend on the values of the cell and its immediate neighbours. Following the idea of the MPFA O-scheme, the most natural strategy is to build some subcells whose vertices are the cell centre, a cell vertex, and some additional points, chosen thanks to suitable properties with respect to the location of the discontinuities of $\Lambda$. This is the purpose of the definition of the so-called "harmonic averaging points".

Using the notations of Section 3.1, we furthermore assume that, for all $s \in \mathcal{V}_{K}$, there exist exactly three faces in $\mathcal{F}_{K}$ and three edges in $\mathcal{E}_{K}$ having $\boldsymbol{s}$ as a vertex, and for all $e \in \mathcal{E}_{K}$, there exist exactly two faces in $\mathcal{F}_{K}$ having $e$ as an edge.

\subsection{Harmonic averaging points}

In this section, we recall the construction of the so-called harmonic averaging points, presented in [6]. The idea is to be able, as in the isotropic case, to express the value of $u$ at some point of the interface between two control volumes as a convex combination of the two values of $u$ at the control volume centres. In the case of an homogeneous medium, this point is simply the intersection between the segment formed by the two cell centres and their interface, and the value of $u$ is its linear interpolation. In the case of an heterogeneous isotropic medium, this point is obtained by a harmonic averaging procedure, using (4.2) below, and the value of $u$ is also the harmonic average of the values of $u$ at the two cell centres, using (4.4). Let us then see how we may obtain such a point in the heterogeneous anisotropic case. Consider two domains $K$ and $L$ of $\mathbb{R}^{3}$ with different diffusion matrices (or permeabilities) $\Lambda_{K}$ and $\Lambda_{L}$, separated by a planar interface $\sigma$ as depicted in Figure 3, and let $\boldsymbol{x}_{K} \in K$ and $\boldsymbol{x}_{L} \in L$. Let $d_{K, \sigma}$ (resp. $d_{L, \sigma}$ ) denote the orthogonal distance from $x_{K}$ (resp. $x_{L}$ ) to $\sigma$. We seek some point $\boldsymbol{y}_{\sigma}$ in $\sigma$ where there exists $\alpha_{K, \sigma}>0$ and $\alpha_{L, \sigma}>0$ with $\alpha_{K, \sigma}+\alpha_{L, \sigma}=1$ such that the relation $u\left(\boldsymbol{y}_{\sigma}\right)=\alpha_{K, \sigma} u\left(\boldsymbol{x}_{K}\right)+\alpha_{L, \sigma} u\left(\boldsymbol{x}_{L}\right)$, holds for any function $u$ such that:

(1) $u$ is affine in both $K$ and $L$;

(2) $u$ is continuous on $\sigma=\partial K \cap \partial L$; 


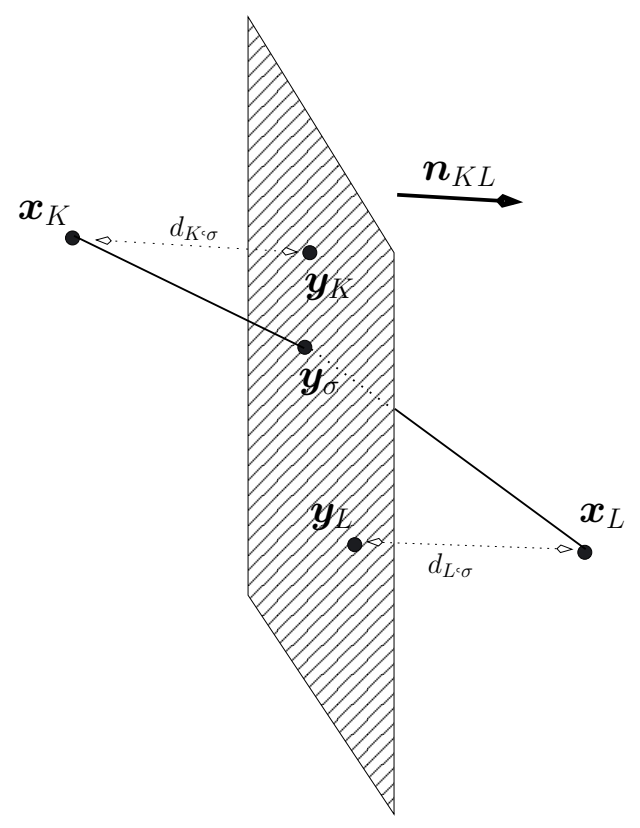

Figure 3. Construction of a particular point at the interface.

(3) the relation

$$
\Lambda_{K} \nabla u\left(\boldsymbol{x}_{K}\right) \cdot \boldsymbol{n}_{K L}+\Lambda_{L} \nabla u\left(\boldsymbol{x}_{L}\right) \cdot \boldsymbol{n}_{L K}=0
$$

holds, where $\boldsymbol{n}_{K L}$ denotes the unit vector, normal to $\sigma$, oriented from $K$ to $L$.

Let $\mathcal{A}_{\sigma, \Lambda}$ denote the set of all functions which satisfy the three above properties; notice that any function $u \in \mathcal{A}_{\sigma, \Lambda}$ is solution to a non homogeneous diffusion problem $-\operatorname{div}(\Lambda(\boldsymbol{x}) \nabla u(\boldsymbol{x}))=0$ for a.e. $\boldsymbol{x} \in K \cup L$.

Therefore, the sought point $\boldsymbol{y}_{\sigma}$ exists and is given by the following formula (see $[6]$ ):

$$
\boldsymbol{y}_{\sigma}=\frac{\lambda_{L} d_{K, \sigma} \boldsymbol{y}_{L}+\lambda_{K} d_{L, \sigma} \boldsymbol{y}_{K}+d_{K, \sigma} d_{L, \sigma}\left(\boldsymbol{\lambda}_{L}^{\sigma}-\boldsymbol{\lambda}_{K}^{\sigma}\right)}{\lambda_{L} d_{K, \sigma}+\lambda_{K} d_{L, \sigma}},
$$

where, denoting by $\mathcal{P}(\boldsymbol{x}, \sigma)$ the orthogonal projection on $\sigma$ of any point $\boldsymbol{x}$, we set

$$
\begin{array}{lll}
\boldsymbol{y}_{K}=\mathcal{P}\left(\boldsymbol{x}_{K}, \sigma\right), & \lambda_{K}=\boldsymbol{n}_{K L} \cdot \Lambda_{K} \boldsymbol{n}_{K L}, & \boldsymbol{\lambda}_{K}^{\sigma}=\Lambda_{K} \boldsymbol{n}_{K L}-\lambda_{K} \boldsymbol{n}_{K L}, \\
\boldsymbol{y}_{L}=\mathcal{P}\left(\boldsymbol{x}_{L}, \sigma\right), & \lambda_{L}=\boldsymbol{n}_{K L} \cdot \Lambda_{L} \boldsymbol{n}_{K L}, & \boldsymbol{\lambda}_{L}^{\sigma}=\Lambda_{L} \boldsymbol{n}_{K L}-\lambda_{L} \boldsymbol{n}_{K L} .
\end{array}
$$

Then the following averaging formula holds:

$$
u\left(\boldsymbol{y}_{\sigma}\right)=\frac{\lambda_{L} d_{K, \sigma} u\left(\boldsymbol{x}_{L}\right)+\lambda_{K} d_{L, \sigma} u\left(\boldsymbol{x}_{K}\right)}{\lambda_{L} d_{K, \sigma}+\lambda_{K} d_{L, \sigma}}, \quad \forall u \in \mathcal{A}_{\sigma, \Lambda}
$$

The verification of this relation relies on the fact that the set of all functions $u \in \mathcal{A}_{\sigma, \Lambda}$ whose values at $\boldsymbol{x}_{K}$ and $\boldsymbol{x}_{L}$ are imposed is an affine set of dimension 2 ; indeed, the degrees of freedom of such a function $u$ are for instance the two components of the gradient $\boldsymbol{g}^{\sigma}$ of the restriction of $u$ to $\sigma$. We can then show that

$$
u(\boldsymbol{y})=\frac{\lambda_{L} d_{K, \sigma} u\left(\boldsymbol{x}_{L}\right)+\lambda_{K} d_{L, \sigma} u\left(\boldsymbol{x}_{K}\right)}{\lambda_{L} d_{K, \sigma}+\lambda_{K} d_{L, \sigma}}+\boldsymbol{g}^{\sigma} \cdot\left(\boldsymbol{y}-\boldsymbol{y}_{\sigma}\right), \quad \forall \boldsymbol{y} \in \sigma,
$$

which shows (4.4). 


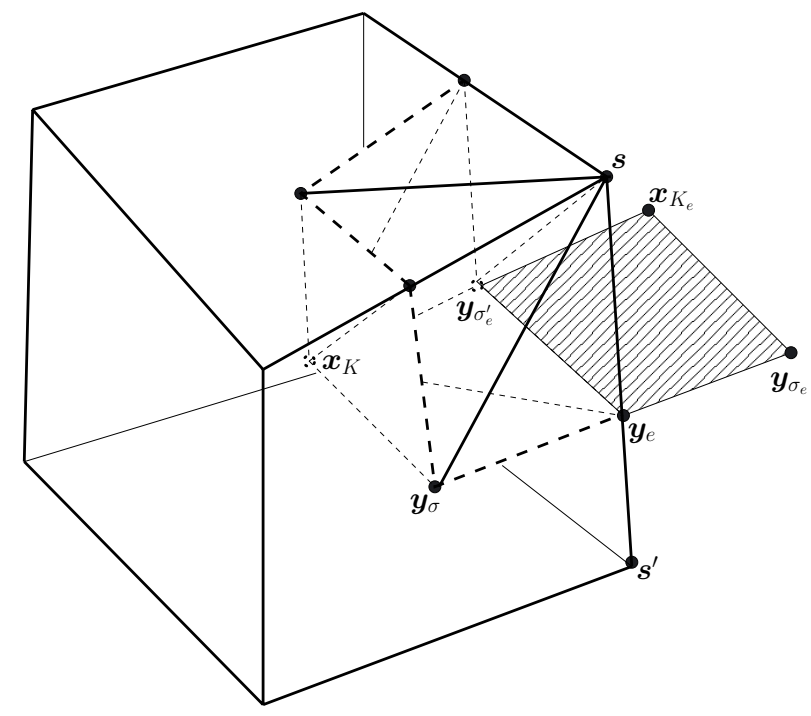

Figure 4. Construction of the submesh $M_{K, s}$ of a control volume.

\subsection{Definition of the scheme}

As in the case of the vertex scheme, we build a piecewise constant discrete gradient. Let us first define a submesh $\left(M_{K, s}\right)_{K \in \mathcal{M}, s \in \mathcal{V}_{K}}$ by the following steps:

(1) define harmonic averaging points in the faces $\sigma \in \mathcal{F}$;

(2) define the "centres" of the edges of the mesh $\left(\boldsymbol{y}_{e}\right)_{e \in \mathcal{E}}$;

(3) define the subcells $M_{K, s}$ of the submesh.

Let us now describe in details each step, all of them referring to Figure 4.

\subsubsection{Definition of harmonic averaging points on the faces}

Since we do not assume the faces to be planar, the construction of the harmonic averaging points on the faces are somewhat more tricky than in the $2 \mathrm{D}$ case. Let us denote $\boldsymbol{x}_{\sigma}$ the barycentre of the vertices $\boldsymbol{s} \in \mathcal{V}_{\sigma}$, and $\boldsymbol{n}_{\sigma}$ an average unit vector normal to $\sigma$ (it can be defined as the average of normal vectors to all triangles defined by $\boldsymbol{x}_{\sigma}$ and two consecutive vertices $\boldsymbol{s}, \boldsymbol{s}^{\prime}$ of $\sigma$ ). Let $\sigma \in \mathcal{F}$ be an interface between the two control volumes $K$ and $L$. We consider the plane $P_{\sigma}$, containing the point $\boldsymbol{x}_{\sigma}$, orthogonal to $\boldsymbol{n}_{\sigma}$. We assume that the geometry of the mesh is sufficiently regular, so that:

(1) the two points $\boldsymbol{x}_{K}$ and $\boldsymbol{x}_{L}$ are not on the same side of $P_{\sigma}$;

(2) the harmonic averaging point $\boldsymbol{y}_{\sigma} \in P_{\sigma}$, defined by (4.2), is such that, denoting by $\boldsymbol{s}_{i}, i=1, \ldots, N_{\sigma}$ the ordered vertices of $\sigma$ (setting $j=i+1$ for $i<N_{\sigma}$ and $j=1$ for $i=N_{\sigma}$, the determinant of the three vectors $\boldsymbol{s}_{i}-\boldsymbol{y}_{\sigma}, \boldsymbol{s}_{j}-\boldsymbol{y}_{\sigma}, \boldsymbol{n}_{\sigma}$ is strictly positive) the determinants of the three vectors $\boldsymbol{s}_{i}-\boldsymbol{y}_{\sigma}, \boldsymbol{s}_{j}-\boldsymbol{y}_{\sigma}$ and $\boldsymbol{x}_{K}-\boldsymbol{y}_{\sigma}$ have all the same sign, opposite to that of the determinants of the three vectors $\boldsymbol{s}_{i}-\boldsymbol{y}_{\sigma}$, $\boldsymbol{s}_{j}-\boldsymbol{y}_{\sigma}$ and $\boldsymbol{x}_{L}-\boldsymbol{y}_{\sigma}$.

The second assumption may not be satisfied in practice in case of a large aspect ratio or large anisotropy ratio. In this case, it is advisable to add a face unknown, as was done in the $2 \mathrm{D}$ case [6]. In Table 5 below, we show the number of face unknowns (the so-called hybrid face unknowns) that were added during the implementation the scheme. 
We then denote by $\alpha_{K, \sigma}$ and $\alpha_{L, \sigma}$ the two coefficients issued from (4.4):

$$
\alpha_{K, \sigma}=\frac{\lambda_{K} d_{L, \sigma}}{\lambda_{L} d_{K, \sigma}+\lambda_{K} d_{L, \sigma}}, \quad \alpha_{L, \sigma}=\frac{\lambda_{L} d_{K, \sigma}}{\lambda_{L} d_{K, \sigma}+\lambda_{K} d_{L, \sigma}} .
$$

\subsubsection{Definition of the "edge centres"}

For a given edge $e \in \mathcal{E}$, we consider the subset $\mathcal{M}_{e}$ of all $K \in \mathcal{M}$, such that $e \in \mathcal{E}_{K}$. For any $K \in \mathcal{M}_{e}$, let $\sigma$ and $\sigma^{\prime}$ be the two faces of $K$ such that $e$ is an edge of $\sigma$ and $\sigma^{\prime}$. Assuming that the points $\boldsymbol{x}_{K}, \boldsymbol{y}_{\sigma}$ and $\boldsymbol{y}_{\sigma^{\prime}}$ are not aligned (this is the case for any reasonable mesh), we can consider the unique plane containing the three points $\boldsymbol{x}_{K}, \boldsymbol{y}_{\sigma}$ and $\boldsymbol{y}_{\sigma^{\prime}}$ : its intersection with the straight line containing $e$ is exactly equal to a unique point, denoted $\boldsymbol{y}_{K, e}$. We denote by $K_{e}$ an element of $\mathcal{M}_{e}$, such that the point $\boldsymbol{y}_{K_{e}, e}$ is the closest to the middle of $e$. We then assume that $\boldsymbol{y}_{K_{e}, e}$, denoted $\boldsymbol{y}_{e}$ for simple and called the "centre" of $e$, belongs to $e$ (see Fig. 4).

The corresponding faces $\sigma$ and $\sigma^{\prime}$ are then denoted $\sigma_{e}$ and $\sigma_{e}^{\prime}$, to which we associate two coefficients $\beta_{e}$ and $\beta_{e}^{\prime}$ such that:

$$
\boldsymbol{y}_{e}=\boldsymbol{x}_{K_{e}}+\beta_{e}\left(\boldsymbol{y}_{\sigma_{e}}-\boldsymbol{x}_{K_{e}}\right)+\beta_{e}^{\prime}\left(\boldsymbol{y}_{\sigma_{e}^{\prime}}-\boldsymbol{x}_{K_{e}}\right) .
$$

\subsubsection{Definition of the submesh}

Let us now consider, for $K \in \mathcal{M}$ and $s \in \mathcal{V}_{K}$, the three faces $\sigma_{i}, i=1,2,3$ and the three edges $e_{i}, i=1,2,3$ having $s$ as a vertex. We assume that $e_{i}$ is a common edge to the faces $\sigma_{i}$ and $\sigma_{j}$, where $j=i+1$ for $i=1,2$ and $j=1$ for $i=3$. We then define $M_{K, s}$ as the polyhedron such that:

(1) its vertices are $\boldsymbol{x}_{K}, \boldsymbol{s}, \boldsymbol{y}_{\sigma_{i}}$ and $\boldsymbol{y}_{e_{i}}$, for $i=1,2,3$;

(2) its "hybrid" faces (which will lead to the definition of hybrid unknowns in the sense of [21]) are the triangles with vertices $\boldsymbol{s}, \boldsymbol{y}_{\sigma_{i}}, \boldsymbol{y}_{e_{i}}$ and $\boldsymbol{s}, \boldsymbol{y}_{\sigma_{j}}, \boldsymbol{y}_{e_{i}}$, where $j=i+1$ for $i=1,2$ and $j=1$ for $i=3$; the set of these 6 triangular faces is denoted by $\widehat{\mathcal{F}}_{K, \boldsymbol{s}}$; we then denote by $\boldsymbol{y}_{\widehat{\tau}}$ the centre of gravity of such a triangular face $\widehat{\tau}$;

(3) its "barycentric" faces (which will lead to the definition of barycentric unknowns in the sense of [21]) are the triangles with vertices $\boldsymbol{x}_{K}, \boldsymbol{y}_{\sigma_{i}}, \boldsymbol{y}_{e_{i}}$ and $\boldsymbol{x}_{K}, \boldsymbol{y}_{\sigma_{j}}, \boldsymbol{y}_{e_{i}}$, where $j=i+1$ for $i=1,2$ and $j=1$ for $i=3$; the set of these 6 triangular faces is denoted by $\widetilde{\mathcal{F}}_{K, \boldsymbol{s}}$; we then denote by $\boldsymbol{y}_{\widetilde{\tau}}$ the centre of gravity of such a triangular face $\widetilde{\tau}$.

It results from this definition that the volume $M_{K, s}$ has 8 vertices and 12 triangular faces. We finally define the set $\mathcal{F}_{K, s}=\widehat{\mathcal{F}}_{K, s} \cup \widetilde{\mathcal{F}}_{K, s}$.

\subsubsection{Definition of the discrete unknown space}

Setting $\widehat{\mathcal{F}}=\bigcup_{K \in \mathcal{M}, s \in \mathcal{V}_{K}} \widehat{\mathcal{F}}_{K, \boldsymbol{s}}$ and $\widetilde{\mathcal{F}}=\bigcup_{K \in \mathcal{M}, \boldsymbol{s} \in \mathcal{V}_{K}} \widetilde{\mathcal{F}}_{K, \boldsymbol{s}}$, we define the set $X_{\mathcal{D}, 0}$ as the set of all real families $u=\left(\left(u_{K}\right)_{K \in \mathcal{M}},\left(u_{\sigma}\right)_{\sigma \in \mathcal{F}},\left(u_{e}\right)_{e \in \mathcal{E}},\left(u_{\widehat{\tau}}\right)_{\widehat{\tau} \in \widehat{\mathcal{F}}},\left(u_{\tilde{\tau}}\right)_{\widetilde{\tau} \in \tilde{\mathcal{F}}}\right)$ such that:

(1) for all exterior face $\sigma \in \mathcal{F}, u_{\sigma}=0$, for all exterior face $\widehat{\tau} \in \widehat{\mathcal{F}}, u_{\widehat{\tau}}=0$, for all exterior edge $e \in \mathcal{E}, u_{e}=0$;

(2) for all interior face $\sigma \in \mathcal{F}$, interface between the two control volumes $K$ and $L$, using (4.5),

$$
u_{\sigma}=\alpha_{K, \sigma} u_{K}+\alpha_{L, \sigma} u_{L}
$$

(3) for all interior edge $e \in \mathcal{E}$, and $K_{e}$ defined as in Section 4.2.2. Then, using (4.6),

$$
u_{e}=u_{K_{e}}+\beta_{e}\left(u_{\sigma_{e}}-u_{K_{e}}\right)+\beta_{e}^{\prime}\left(u_{\sigma_{e}^{\prime}}-u_{K_{e}}\right)
$$

(4) for all "barycentric" triangular face $\widetilde{\tau} \in \widetilde{\mathcal{F}}$ with vertices $\boldsymbol{x}_{K}, \boldsymbol{y}_{\sigma}, \boldsymbol{y}_{e}$ set

$$
u_{\widetilde{\tau}}=\frac{1}{3}\left(u_{K}+u_{\sigma}+u_{e}\right)
$$


Hence the degrees of freedom of $X_{\mathcal{D}, 0}$ are the values $u_{K}$, for $K \in \mathcal{M}$, and the values $u_{\sigma}$, for $\sigma \in \widehat{\mathcal{F}}_{\text {int }}$, which are the interior faces of $\widehat{\mathcal{F}}$.

\subsubsection{Definition of an approximate gradient and of a reconstruction operator}

We first define, for all $u \in X_{\mathcal{D}, 0}$, for all $K \in \mathcal{M}$ and $s \in \mathcal{V}_{K}$ :

$$
\nabla_{K, \boldsymbol{s}} u=\frac{1}{\left|M_{K, \boldsymbol{s}}\right|} \sum_{\tau \in \mathcal{F}_{K, \boldsymbol{s}}}|\tau|\left(u_{\tau}-u_{K}\right) \boldsymbol{n}_{K, \boldsymbol{s}}^{\tau}
$$

where $\boldsymbol{n}_{K, \boldsymbol{s}}^{\tau}$ is the unit normal vector, outward to $M_{K, \boldsymbol{s}}$, to the triangular face $\tau$. As in the vertex centred case, this expression yields a consistent expression of the gradient. In order to enhance the stability of this gradient, we define

and

$$
R_{K, \boldsymbol{s}}^{\tau} u=u_{\tau}-u_{K}-\nabla_{K, \boldsymbol{s}} u \cdot\left(\boldsymbol{y}_{\tau}-\boldsymbol{x}_{K}\right), \forall \tau \in \mathcal{F}_{K, \boldsymbol{s}}
$$

$$
\nabla_{K, s}^{\tau} u=\nabla_{K, s} u+\gamma R_{K, s}^{\tau} u \frac{|\tau|}{\left|M_{K, \boldsymbol{s}}\right|} \boldsymbol{n}_{K, \boldsymbol{s}}^{\tau},
$$

where $\gamma>0$ is a positive coefficient, whose standard value is 1 in the numerical tests. We then denote by $\left(M_{K, s}^{\tau}\right)_{\tau \in \mathcal{F}_{K, s}}$ a partition of $M_{K, s}$ such that $\left|M_{K, s}^{\tau}\right|=\left|M_{K, \boldsymbol{s}}\right| / N_{K, \boldsymbol{s}}$, where $N_{K, \boldsymbol{s}}$ is the number of elements of $\mathcal{F}_{K, s}$; note that again, this partition does not need to be defined precisely. We then define $\nabla_{\mathcal{D}} u$ by the constant value $\nabla_{K, s}^{\tau} u$ in $M_{K, s}^{\tau}$.

As in Section 3, the piecewise constant reconstruction $\Pi_{\mathcal{D}}^{0} v$ is defined as the function equal to $v_{K}$ in each $K$, and the piecewise affine reconstruction is defined by $\Pi_{\mathcal{D}} u(\boldsymbol{x})=u_{K}+\nabla_{K, \boldsymbol{s}} u \cdot\left(\boldsymbol{x}-\boldsymbol{x}_{K}\right)$ for a.e. $\boldsymbol{x} \in M_{K, \boldsymbol{s}}$ for all $K \in \mathcal{M}$.

\subsubsection{Definition and implementation of the scheme}

Problem (1.1) is again approximated by (1.2), which can also be written in the framework of this scheme

$$
\begin{aligned}
& \text { find } u \in X_{\mathcal{D}, 0}, \forall v \in X_{\mathcal{D}, 0}, \\
& \sum_{K \in \mathcal{M}} \sum_{\boldsymbol{s} \in \mathcal{V}_{K}} \sum_{\tau \in \mathcal{F}_{K, \boldsymbol{s}}}\left|M_{K, \boldsymbol{s}}^{\tau}\right| \Lambda_{K} \nabla_{K, \boldsymbol{s}}^{\tau} u \cdot \nabla_{K, \boldsymbol{s}}^{\tau} v=\sum_{K \in \mathcal{M}} \sum_{\boldsymbol{s} \in \mathcal{V}_{K}} \int_{M_{K, \boldsymbol{s}}}\left(v_{K}+\nabla_{K, \boldsymbol{s}} v \cdot\left(\boldsymbol{x}-\boldsymbol{x}_{K}\right)\right) f(\boldsymbol{x}) \mathrm{d} \boldsymbol{x} .
\end{aligned}
$$

Let us now detail the equations resulting from scheme (4.10).

We first define the linear forms $F_{K, s}^{\tau}$, only depending on $\left(u_{\tau^{\prime}}-u_{K}\right)_{\tau^{\prime} \in \widehat{\mathcal{F}}_{K, s}}$, such that the relation

$$
\sum_{\boldsymbol{s} \in \mathcal{V}_{K}} \sum_{\tau \in \mathcal{F}_{K, \boldsymbol{s}}}\left|M_{K, \boldsymbol{s}}^{\tau}\right| \Lambda_{K} \nabla_{K, \boldsymbol{s}}^{\tau} u \cdot \nabla_{K, \boldsymbol{s}}^{\tau} v=\sum_{\tau \in \mathcal{F}_{K, \boldsymbol{s}}} F_{K, \boldsymbol{s}}^{\tau} u\left(v_{K}-v_{\tau}\right)
$$

holds for all real values $\left(u_{\tau^{\prime}}-u_{K}\right)_{\tau^{\prime} \in \mathcal{F}_{K, s}}$ and $\left(v_{\tau^{\prime}}-v_{K}\right)_{\tau^{\prime} \in \mathcal{F}_{K, s}}$. Assume that $\widehat{\tau}$ is a common face to $M_{K, \boldsymbol{s}}$ and $M_{L, s}$. Then the equation, resulting from setting $v_{\widehat{\tau}}=1$ and all other degrees of freedom equal to 0 , is

$$
F_{K, s}^{\widehat{\tau}} u+F_{L, s}^{\widehat{\tau}} u=\rho^{\widehat{\tau}}(f),
$$

where

$$
\rho^{\widehat{\tau}}(f)=\frac{1}{\left|M_{K, \boldsymbol{s}}\right|} \int_{M_{K, \boldsymbol{s}}}|\tau| \boldsymbol{n}_{K, \boldsymbol{s}}^{\tau} \cdot\left(\boldsymbol{x}-\boldsymbol{x}_{K}\right) f(\boldsymbol{x}) \mathrm{d} \boldsymbol{x}+\frac{1}{\left|M_{L, \boldsymbol{s}}\right|} \int_{M_{L, \boldsymbol{s}}}|\tau| \boldsymbol{n}_{L, \boldsymbol{s}}^{\tau} \cdot\left(\boldsymbol{x}-\boldsymbol{x}_{L}\right) f(\boldsymbol{x}) \mathrm{d} \boldsymbol{x} .
$$

Note that if we use $\Pi_{\mathcal{D}}^{0}$ instead of $\Pi_{\mathcal{D}}, \rho^{\widehat{\tau}}(f)=0$ and therefore the flux conservation of the external faces is ensured. Since there are as many equations (4.12), as there are triangular faces having $s$ as a vertex, it is 
possible to eliminate all the unknowns $u_{\widehat{\tau}}$, expressing them with respect to all $\left(u_{K}\right)_{K \in \mathcal{M}_{s}}$, where $\mathcal{M}_{\boldsymbol{s}}$ denotes, for a given $s \in \mathcal{V}$, the subset of all $K \in \mathcal{M}$ such that $s \in \mathcal{V}_{K}$. We indeed show in the next section that the system (4.12) is invertible. Let us now study the stencil of the scheme. Using the definition of the space $X_{\mathcal{D}, 0}$, due to the definition of harmonic averaging values at the faces and due to the interpolation $u_{e}$ at the point $\boldsymbol{y}_{e}$, we can write, for $v \in X_{\mathcal{D}, 0}$ and for a given triangular face $\widetilde{\tau} \in \widetilde{\mathcal{F}}_{K, \boldsymbol{s}}$,

$$
v_{\widetilde{\tau}}=\sum_{L \in \mathcal{M}_{e}} \beta_{\widetilde{\tau}, L} v_{L}
$$

where $e \in \mathcal{E}$ is an edge such that $\boldsymbol{y}_{e}$ is a vertex of $\widetilde{\tau}$, and where we denote $\mathcal{M}_{e}$ the set of all $L \in \mathcal{M}$ such that $e \in \mathcal{E}_{L}$. Note that the condition

$$
\sum_{L \in \mathcal{M}_{e}} \beta_{\widetilde{\tau}, L}=1
$$

holds in the case where the control volume $K$ has no vertex on the boundary of the domain. An important consequence is that, if $\mathcal{V}_{e}=\left\{s, s^{\prime}\right\}$ (see Fig. 4), then

$$
\mathcal{M}_{e} \subset \mathcal{M}_{s} \cap \mathcal{M}_{s^{\prime}}
$$

We then set $\beta_{\widetilde{\tau}, L}=0$ for all $L \in\left(\mathcal{M}_{\boldsymbol{s}} \cup \mathcal{M}_{\boldsymbol{s}^{\prime}}\right) \backslash \mathcal{M}_{e}$.

It results from this relation that the equation, obtained by setting $v_{K}=1$ in (4.10) and all other degrees of freedom equal to 0 , reads

$$
\sum_{s \in \mathcal{V}_{K}}\left(\sum_{\widehat{\tau} \in \hat{\mathcal{F}}_{K, s}} F_{K, s}^{\widehat{\tau}} u+\sum_{\widetilde{\tau} \in \widetilde{\mathcal{F}}_{K, s}} F_{K, s}^{\widetilde{\tau}} u-\sum_{L \in \mathcal{M}_{s}} \sum_{\widetilde{\tau} \in \widetilde{\mathcal{F}}_{L, s}} \beta_{\widetilde{\tau}, K} F_{L, s}^{\widetilde{\tau}} u\right)=\rho_{K}(f),
$$

where

$$
\rho_{K}(f)=\int_{K} f(\boldsymbol{x}) \mathrm{d} x-\sum_{\boldsymbol{s} \in \mathcal{V}_{K}} \sum_{\widehat{\tau} \in \widehat{\mathcal{F}}_{K, \boldsymbol{s}}} \frac{1}{\left|M_{K, \boldsymbol{s}}\right|} \int_{M_{K, \boldsymbol{s}}}|\tau| \boldsymbol{n}_{K, \boldsymbol{s}}^{\tau} \cdot\left(\boldsymbol{x}-\boldsymbol{x}_{K}\right) f(\boldsymbol{x}) \mathrm{d} \boldsymbol{x} .
$$

Note that if we use $\Pi_{\mathcal{D}}^{0}$ instead of $\Pi_{\mathcal{D}}$, we get $\rho_{K}(f)=\int_{K} f(\boldsymbol{x}) \mathrm{d} x$. From (4.14), we get that (4.10) is indeed a symmetric 27-point stencil scheme in the case of a structured mesh.

\subsection{Mathematical analysis}

Lemma 4.1 (gradient scheme properties and invertibility of the local systems). Let $\mathcal{D}=\left(X_{\mathcal{D}, 0}, h_{\mathcal{D}}, \Pi_{\mathcal{D}}, \nabla_{\mathcal{D}}\right)$ be defined in the present Section 4, then, for a fixed $\gamma$, the scheme (1.2) is a gradient scheme in the sense of Definition 2.1, and therefore there exists a unique solution to (1.2). Furthermore, the local systems (4.12) are invertible.

Proof. The proof of this lemma follows the first steps of the proof of Lemma 3.1. We first remark that, for any $u \in X_{\mathcal{D}, 0}$,

$$
\int_{\Omega}\left|\nabla_{\mathcal{D}} u(\boldsymbol{x})\right|^{2} \mathrm{~d} \boldsymbol{x}=\sum_{K \in \mathcal{M}} \sum_{\boldsymbol{s} \in \mathcal{V}_{K}} \sum_{\tau \in \mathcal{F}_{K, \boldsymbol{s}}} \frac{\left|M_{K, \boldsymbol{s}}\right|}{N_{K, \boldsymbol{s}}}\left|\nabla_{K, \boldsymbol{s}}^{\tau} u\right|^{2} .
$$

The following property (which is similar to (3.9)) holds

$$
\begin{aligned}
\sum_{\tau \in \mathcal{F}_{K, \boldsymbol{s}}} R_{K, \boldsymbol{s}}^{\tau} u \frac{|\tau|}{\left|M_{K, \boldsymbol{s}}\right|} \boldsymbol{n}_{K, \boldsymbol{s}}^{\tau} & =\sum_{\tau \in \mathcal{F}_{K, \boldsymbol{s}}}\left(\left(u_{\tau}-u_{K}\right) \frac{|\tau|}{\left|M_{K, \boldsymbol{s}}\right|} \boldsymbol{n}_{K, \boldsymbol{s}}^{\tau}-\frac{|\tau|}{\left|M_{K, \boldsymbol{s}}\right|}\left(\boldsymbol{y}_{\tau}-\boldsymbol{x}_{K}\right)\left(\boldsymbol{n}_{K, \boldsymbol{s}}^{\tau}\right)^{t} \nabla_{K, \boldsymbol{s}} u\right) \\
& =\nabla_{K, \boldsymbol{s}} u-\nabla_{K, \boldsymbol{s}} u=0 .
\end{aligned}
$$


It implies

$$
\sum_{\tau \in \mathcal{F}_{K, s}} \frac{\left|M_{K, \boldsymbol{s}}\right|}{N_{K, \boldsymbol{s}}}\left|\nabla_{K, \boldsymbol{s}}^{\tau} u\right|^{2}=\left|M_{K, \boldsymbol{s}}\right|\left|\nabla_{K, \boldsymbol{s}} u\right|^{2}+\sum_{\tau \in \mathcal{F}_{K, s}} \frac{\left|M_{K, \boldsymbol{s}}\right|}{N_{K, \boldsymbol{s}}}\left(\gamma R_{K, \boldsymbol{s}}^{\tau} u \frac{|\tau|}{\left|M_{K, \boldsymbol{s}}\right|}\right)^{2} .
$$

As in the proof of Lemma 3.1, we again have, for a value $\mu_{K, s}^{\tau}$ chosen later, that

$$
\left(R_{K, \boldsymbol{s}}^{\tau} u\right)^{2} \geq \frac{\mu_{K, \boldsymbol{s}}^{\tau}}{1+\mu_{K, \boldsymbol{s}}^{\tau}}\left(u_{\tau}-u_{K}\right)^{2}-\mu_{K, \boldsymbol{s}}^{\tau}\left|\nabla_{K, \boldsymbol{s}} u\right|^{2}\left|\boldsymbol{y}_{\tau}-\boldsymbol{x}_{K}\right|^{2} .
$$

We define $\mu_{K, \boldsymbol{s}}^{\tau}>0$ by

$$
1-\mu_{K, \boldsymbol{s}}^{\tau} \gamma^{2}\left|\boldsymbol{y}_{\tau}-\boldsymbol{x}_{K}\right|^{2}\left(\frac{|\tau|}{\left|M_{K, \boldsymbol{s}}\right|}\right)^{2}=0, \forall K \in \mathcal{M}_{\boldsymbol{s}}
$$

This proves, introducing $C_{\mu}$ as the maximum value of all $\mu_{K, s}^{\tau}$,

$$
\int_{\Omega}\left|\nabla_{\mathcal{D}} u(\boldsymbol{x})\right|^{2} \mathrm{~d} \boldsymbol{x} \geq \frac{1}{1+C_{\mu}}\|u\|_{1, \mathcal{D}}^{2}
$$

where:

$$
\|u\|_{1, \mathcal{D}}^{2}=\sum_{K \in \mathcal{M}} \sum_{\boldsymbol{s} \in \mathcal{V}_{K}} \sum_{\tau \in \mathcal{F}_{K, \boldsymbol{s}}} \frac{\left|M_{K, \boldsymbol{s}}\right|}{N_{K, \boldsymbol{s}}} \frac{\left(u_{\tau}-u_{K}\right)^{2}}{\left|\boldsymbol{y}_{\tau}-\boldsymbol{x}_{K}\right|^{2}}
$$

A first consequence of (4.17) is that the local system of equations (4.12), written for all $\widehat{\tau} \in \bigcup_{K \in \mathcal{M}_{s}} \widehat{\mathcal{F}}_{K, \boldsymbol{s}}$, whose unknowns are the values $u_{\widehat{\tau}}$, is invertible. Indeed, assume that all $u_{K}=0$ for all $K \in \mathcal{M}$ and $u_{\tau}=0$ for all $\tau \notin \bigcup_{K \in \mathcal{M}_{s}} \widehat{\mathcal{F}}_{K, \boldsymbol{s}}$. We multiply (4.12) by $-u_{\widehat{\tau}}$, and sum the resulting equation on $\widehat{\tau} \in \bigcup_{K \in \mathcal{M}_{s}} \widehat{\mathcal{F}}_{K, \boldsymbol{s}}$. We get

$$
\sum_{K \in \mathcal{M}_{s}} \sum_{\widehat{\tau} \in \widehat{\mathcal{F}}_{K, s}}\left(0-u_{\widehat{\tau}}\right) F_{K, s}^{\widehat{\tau}} u=0
$$

For this particular choice of $u \in X_{\mathcal{D}, 0}$, we get from (4.11) that

$$
\sum_{K \in \mathcal{M}_{\boldsymbol{s}}} \sum_{\widehat{\tau} \in \widehat{\mathcal{F}}_{K, \boldsymbol{s}}}\left(0-u_{\widehat{\tau}}\right) F_{K, \boldsymbol{s}}^{\widehat{\tau}} u=\int_{\Omega} \Lambda(\boldsymbol{x}) \nabla_{\mathcal{D}} u(\boldsymbol{x}) \cdot \nabla_{\mathcal{D}} u(\boldsymbol{x}) \mathrm{d} \boldsymbol{x}
$$

From (4.17), we then prove that $\left(u_{\tau}-u_{K}\right)=0$ for all $K \in \mathcal{M}, s \in \mathcal{V}_{K}$ and $\tau \in \mathcal{F}_{K, \boldsymbol{s}}$. This implies in particular that $u_{\widehat{\tau}}=0$ for all $\widehat{\tau} \in \bigcup_{K \in \mathcal{M}_{s}} \widehat{\mathcal{F}}_{K, \boldsymbol{s}}$, thus proving the invertibility of system (4.12).

A second consequence of $(4.17)$ is that $\left\|\nabla_{\mathcal{D}} \cdot\right\|_{L^{2}(\Omega)^{d}}$ is a norm on $X_{\mathcal{D}, 0}$, which concludes the proof.

This scheme may then be shown to be convergent and exact for piecewise affine solutions with the same tools as those developed in Section 3, thanks to the fact that the barycentric extrapolations involved in the definition of the scheme are all defined through the values taken within the closure of the same cell.

\section{Numerical RESUltS}

In this section, some test cases have been done in order to compare the exact solution and the approximate solutions obtained with MPFA O and L schemes and the new gradient schemes which we denote by VAG for the vertex approximate gradient scheme, and HAG for the harmonic approximate gradient scheme. Several tests are taken from the FVCA Benchmark 3D [23]. Therein additional results on the VAG scheme are presented for a larger family of numerical tests and for several criteria of comparison. 
TABLE 1. $L^{2}$ norm of the error for $\Lambda_{1}$ and randomly distorted Cartesian meshes.

\begin{tabular}{|c|c|c|c|c|}
\hline & Mesh 1 & Mesh 2 & Mesh 3 & Mesh 4 \\
\hline VAG scheme & $4.57 \mathrm{e}-02$ & $1.23 \mathrm{e}-02$ & $2.85 \mathrm{e}-03$ & $7.18 \mathrm{e}-04$ \\
\hline HAG scheme & $7.58 \mathrm{e}-02$ & $2.28 \mathrm{e}-02$ & $7.29 \mathrm{e}-03$ & $1.79 \mathrm{e}-03$ \\
\hline O scheme & $8.04 \mathrm{e}-02$ & $2.30 \mathrm{e}-02$ & $5.31 \mathrm{e}-03$ & $1.38 \mathrm{e}-03$ \\
\hline L scheme & $2.76 \mathrm{e}-02$ & $9.83 \mathrm{e}-03$ & $2.53 \mathrm{e}-03$ & $7.07 \mathrm{e}-04$ \\
\hline
\end{tabular}

TABLE 2. $L^{2}$ norm of the error for $\Lambda_{2}$ and randomly distorted Cartesian meshes.

\begin{tabular}{|c|c|c|c|c|}
\hline & Mesh 1 & Mesh 2 & Mesh 3 & Mesh 4 \\
\hline VAG scheme & $4.59 \mathrm{e}-02$ & $1.24 \mathrm{e}-02$ & $2.91 \mathrm{e}-03$ & $7.39 \mathrm{e}-04$ \\
\hline HAG scheme & $7.81 \mathrm{e}-02$ & $2.46 \mathrm{e}-02$ & $9.76 \mathrm{e}-03$ & $2.77 \mathrm{e}-03$ \\
\hline O scheme & $9.70 \mathrm{e}-01$ & $1.85 \mathrm{e}-01$ & $8.92 \mathrm{e}-01$ & $9.02 \mathrm{e}-01$ \\
\hline L scheme & $2.40 \mathrm{e}-02$ & $9.98 \mathrm{e}-03$ & $4.01 \mathrm{e}-03$ & $1.94 \mathrm{e}-03$ \\
\hline
\end{tabular}

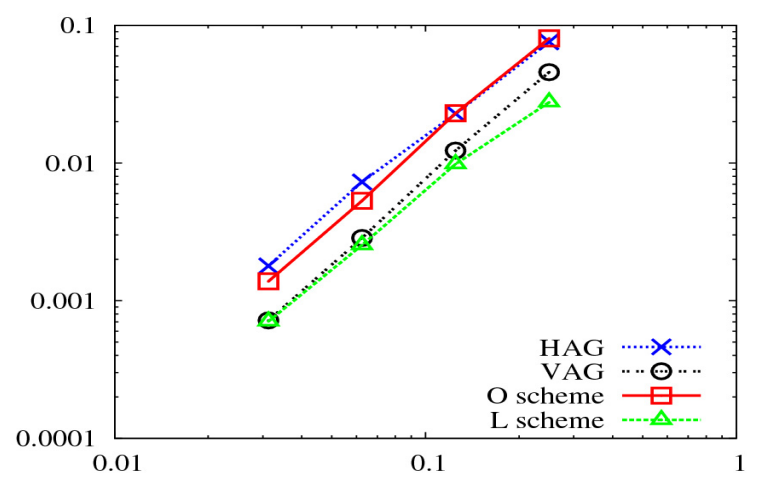

(a) $\Lambda_{1}$

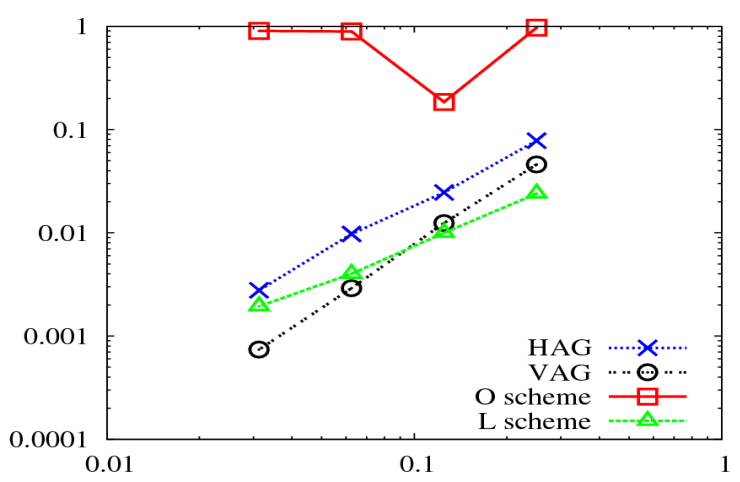

(b) $\Lambda_{2}$

FiguRE 5. $L^{2}$ pressure error function of $h$-randomly distorted Cartesian meshes.

\subsection{Randomly distorted Cartesian meshes}

A family of uniform Cartesian meshes of the domain $\Omega=[0,1]^{3}$ of step size $h$ is considered. A displacement of maximum length $\frac{h}{3}$ is applied in the $x y z$-directions to each node of the Cartesian meshes as exhibited in Figure 6a.

Homogeneous case.. The right hand side $f$ and the Dirichlet boundary condition are such that the exact solution is $u(x, y, z)=\sin (\pi x) \sin (\pi y) \sin (\pi z)$. Two different diffusion tensors $\Lambda_{1}=\operatorname{diag}(1,1,100)$ and $\Lambda_{2}=\operatorname{diag}(1,1$, 1000) are considered. Tables 1 and 2 below exhibit the discrete $L^{2}$ norm of the error between the exact solution and the approximate solutions obtained with MPFA O and L schemes and the new gradient schemes.

In Figure 5 the $L^{2}$ norm of the error is plotted as a function of $h$. Observe first that the smallest errors are obtained with the L scheme or the VAG scheme. Comparing Figures 5a and 5b, we clearly see the failure of the O scheme when the anisotropy increases while the L scheme, the VAG scheme and the HAG schemes remain convergent. This is due to the loss of coercivity of the $\mathrm{O}$ scheme when a large anisotropic ratio is combined with a distortion of the mesh. Note that for these tests, harmonic points were found for all the interfaces with the HAG scheme, so that no additional hybrid unknown was required. 


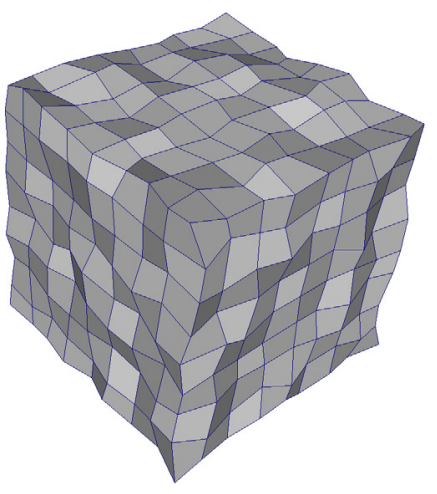

(a) Homogeneous case. 3D view

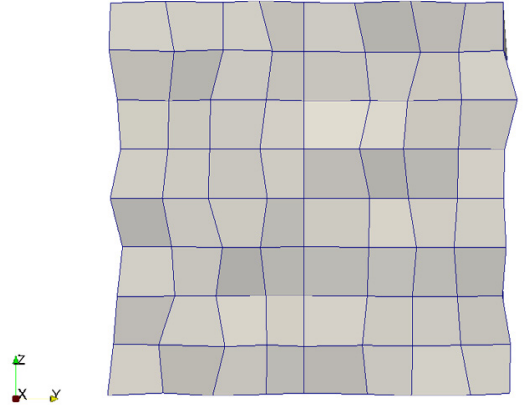

(b) Heterogeneous case. 2D yz-cut

FiguRE 6. Randomly distorted hexahedral mesh for $h=\frac{1}{8}$.

Heterogeneous case.. The test case presented here is described in [4]. We still consider a family of randomly perturbed grids of the domain $\Omega=\Omega_{1} \cup \Omega_{2}$ where $\Omega_{1}=[0,1] \times[0,0.5] \times[0,1]$ and $\Omega_{2}=[0,1] \times[0.5,1] \times[0,1]$. Notice that the perturbation is applied in the $x y$-directions on each nodes except at $y=0.5$ such that the discontinuity is planar as shown in Figure 6b. The right hand side is $f=0$ and the Dirichlet boundary condition are given by the exact solution $u(x, y, z)$ defined by

$$
u(x, y, z)=\left\{\begin{array}{l}
x+y+z \text { in } \Omega_{1}, \\
x-\frac{y}{2}+z+\frac{3}{4} \text { in } \Omega_{2} .
\end{array}\right.
$$

The following permeability tensors $\Lambda_{1}$ and $\Lambda_{2}$ are respectively assigned to the subdomains $\Omega_{1}$ and $\Omega_{2}$,

$$
\Lambda_{1}=\operatorname{diag}(1,1,1) \quad \text { and } \quad \Lambda_{2}=\left(\begin{array}{lll}
2 & 1 & 1 \\
1 & 2 & 1 \\
1 & 1 & 2
\end{array}\right) .
$$

As expected, the VAG and HAG schemes provide the exact solution if $\Lambda$ is piecewise constant in polygonal sub-domains and $u(x, y, z)$ is affine in each of these sub-domains.

\subsection{Near-well meshes}

As we mentioned in the introduction, in reservoir engineering, a proper well modelling requires an accurate multiphase flow simulation which takes into account the singular pressure distribution in the well vicinity and the large difference of scales between the wellbore radius and the reservoir dimension. In this example, we consider the numerical simulation of a single-phase flow in near-well regions for a deviated well. The medium is homogeneous, but anisotropic. An analytical solution of this problem is described in [2] for a diagonal diffusion tensor in the $x y z$-coordinate system. Diagonal elements are denoted as $\Lambda_{x}, \Lambda_{y}$ and $\Lambda_{z}$.

The near-well model proposed here is based on 3D meshes that are refined around the well as illustrated in Figure 1. The first step of the discretization is to create a radial mesh that is exponentially refined down to the well boundary. This radial local refinement requires a matching mesh between the radial grid and the reservoir Cartesian (or "corner point geometry") grid using hexaedra.

Given $\Lambda_{x}=\Lambda_{y}=\eta \Lambda_{z}$, two anisotropy ratios $\eta$ are tested, $\eta=5$ and $\eta=20$. The discrete equation is solved as a Dirichlet problem, with the analytical solution imposes both on the wellbore boundary and the outer boundary. Figure 7 exhibits the discrete solution. It can be noticed that the isobars shade into ellipses away from the circular wellbore. 


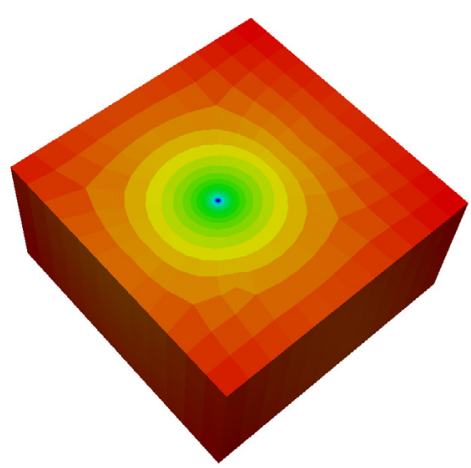

FigURE 7. Solution computed with the near-well mesh 4 and $\eta=5$.

TABLE 3. $L^{2}$ norm of the error for $\eta=5$ and near-well meshes.

\begin{tabular}{|c|c|c|c|c|c|c|c|}
\hline & Mesh 1 & Mesh 2 & Mesh 3 & Mesh 4 & Mesh 5 & Mesh 6 & Mesh 7 \\
\hline VAG scheme & $4.82 \mathrm{e}-03$ & $2.02 \mathrm{e}-03$ & $8.56 \mathrm{e}-04$ & $5.54 \mathrm{e}-04$ & $2.99 \mathrm{e}-04$ & $1.47 \mathrm{e}-04$ & $1.52 \mathrm{e}-04$ \\
\hline HAG scheme & $3.80 \mathrm{e}-03$ & $1.90 \mathrm{e}-03$ & $9.21 \mathrm{e}-04$ & $5.46 \mathrm{e}-04$ & $3.86 \mathrm{e}-04$ & $2.70 \mathrm{e}-04$ & $2.34 \mathrm{e}-04$ \\
\hline O scheme & $7.49 \mathrm{e}-03$ & $3.00 \mathrm{e}-03$ & $1.31 \mathrm{e}-03$ & $7.88 \mathrm{e}-04$ & $4.70 \mathrm{e}-04$ & $2.94 \mathrm{e}-04$ & $2.04 \mathrm{e}-04$ \\
\hline L scheme & $7.34 \mathrm{e}-03$ & $2.95 \mathrm{e}-03$ & $1.33 \mathrm{e}-03$ & $8.03 \mathrm{e}-04$ & $5.00 \mathrm{e}-04$ & $3.44 \mathrm{e}-04$ & $2.34 \mathrm{e}-04$ \\
\hline
\end{tabular}

TABLE 4. $L^{2}$ norm of the error for $\eta=20$ and near-well meshes.

\begin{tabular}{|c|c|c|c|c|c|c|c|}
\hline & Mesh 1 & Mesh 2 & Mesh 3 & Mesh 4 & Mesh 5 & Mesh 6 & Mesh 7 \\
\hline VAG scheme & $5.50 \mathrm{e}-03$ & $2.42 \mathrm{e}-03$ & $1.04 \mathrm{e}-03$ & $6.57 \mathrm{e}-04$ & $3.22 \mathrm{e}-04$ & $1.37 \mathrm{e}-04$ & $1.25 \mathrm{e}-04$ \\
\hline HAG scheme & $4.82 \mathrm{e}-03$ & $2.32 \mathrm{e}-03$ & $1.17 \mathrm{e}-03$ & $6.14 \mathrm{e}-04$ & $3.94 \mathrm{e}-04$ & $2.63 \mathrm{e}-04$ & $2.01 \mathrm{e}-04$ \\
\hline O scheme & $9.25 \mathrm{e}-03$ & $3.71 \mathrm{e}-03$ & $1.63 \mathrm{e}-03$ & $9.66 \mathrm{e}-04$ & $5.69 \mathrm{e}-04$ & $3.55 \mathrm{e}-04$ & $2.44 \mathrm{e}-04$ \\
\hline L scheme & $9.03 \mathrm{e}-03$ & $3.62 \mathrm{e}-03$ & $1.65 \mathrm{e}-03$ & $9.83 \mathrm{e}-04$ & $6.07 \mathrm{e}-04$ & $4.23 \mathrm{e}-04$ & $2.84 \mathrm{e}-04$ \\
\hline
\end{tabular}

TABLE 5. Number of hybrid unknowns introduced by the HAG scheme with near-well meshes.

\begin{tabular}{|c|c|c|c|c|c|c|c|}
\hline & Mesh 1 & Mesh 2 & Mesh 3 & Mesh 4 & Mesh 5 & Mesh 6 & Mesh 7 \\
\hline$\#$ control volumes & 890 & 2232 & 5016 & 11220 & 23210 & 42633 & 74679 \\
\hline$\sharp$ hybrid faces & 0 & 32 & 0 & 0 & 24 & 40 & 44 \\
\hline
\end{tabular}

Tables 3 and 4 below exhibit the error measured in $L^{2}$ norm between the exact solution and the approximate solutions obtained with the MPFA $\mathrm{O}$ and $\mathrm{L}$ schemes and the new gradient schemes.

The new gradient schemes are more accurate than the $\mathrm{O}$ and L schemes, particularly on coarse meshes. For finer meshes, the non planarity of the faces diminishes, and therefore the behaviour of the schemes is more uniform. On coarse meshes, the fact that the VAG and HAG schemes define subcells with triangular subfaces makes them less sensitive to the non planarity problems than the $\mathrm{O}$ and $\mathrm{L}$ schemes.

In Figure 8, the $L^{2}$ norm of the error is plotted as a function of $h$, where $h$ is the diameter of the largest cell in the radial part of meshes. Comparing the Figures $8 \mathrm{a}$ and $8 \mathrm{~b}$, we see that the gradient schemes are more robust when the ratio of anisotropy increases especially on the finest mesh. The rate of convergence is $h^{2}$ for all schemes.

Table 5 below shows that the number of hybrid unknowns introduced by the HAG scheme is negligible compared to the number of the cell-centred unknowns. 


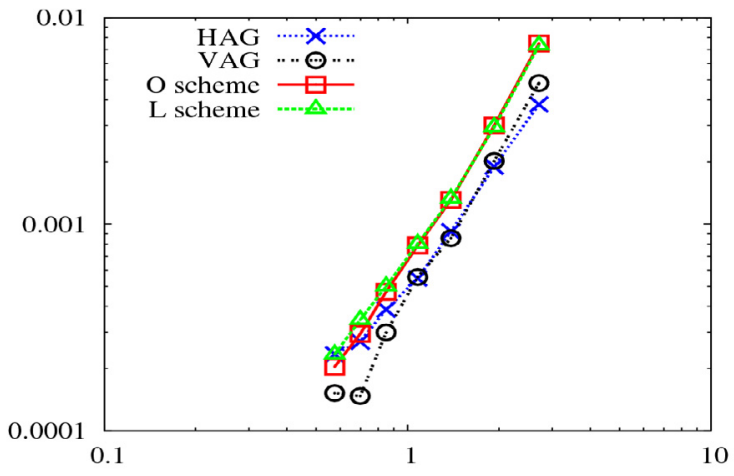

(a) $\eta=5$

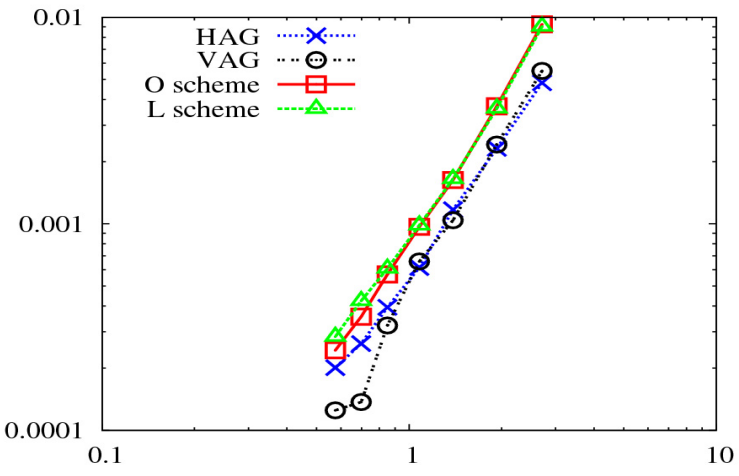

(b) $\eta=20$

FiguRE 8. $L^{2}$ pressure error function of $h$ - near-well meshes.

\section{Conclusion}

In this paper, a simple theoretical framework for the study of gradient schemes was shown to include some classical schemes along with two new schemes which were specially designed to obtain small stencils. Thanks to this theoretical framework, these two schemes are shown to be symmetric, coercive and convergent. A numerical comparison with classical multi-point schemes on realistic industrial 3D cases allows to check the numerical performance of the schemes.

The robustness of such schemes gives them a large advantage over schemes whose coercivity properties are not shown nor numerically verified on some very heterogeneous anisotropic realistic cases. Their symmetry properties allow the use of conjugate gradient methods, whereas BICGStab or GMRES methods are needed in the case of non-symmetric methods. Moreover, their small stencil is adapted to domain decomposition techniques and parallelization.

On going research includes the numerical study of the use of these new schemes for industrial compositional/chemical multi-phase flows in porous media, which occur in reservoir engineering, CO2 storage studies and environmental studies. Recently, we implemented the VAG scheme in the framework of multiphase flow [22] with promising results. This implementation is based on exchange terms between vertices and cells, thus showing that there is no need to identify a real control volume interface to compute the Darcy fluxes.

Acknowledgements. We would like to thank Philippe Angot for interesting discussions and the referees for their useful comments and suggestions.

\section{REFERENCES}

[1] I. Aavatsmark, An introduction to multipoint flux approximations for quadrilateral grids. Comput. Geosci. 6 (2002) 405-432. Locally conservative numerical methods for flow in porous media.

[2] I. Aavatsmark and R. Klausen, Well index in reservoir simulation for slanted and slightly curved wells in 3D grids. SPE J. 8 (2003) 41-48.

[3] I. Aavatsmark, T. Barkve, O. Boe and T. Mannseth, Discretization on non-orthogonal, quadrilateral grids for inhomogeneous, anisotropic media. J. Comput. Phys. 127 (1996) 2-14.

[4] I. Aavatsmark, G. Eigestad, B. Heimsund, B. Mallison, J. Nordbotten and E. Oian, A new finite-volume approach to efficient discretization on challenging grids. SPE J. 15 (2010) 658-669.

[5] L. Agelas, D.A. Di Pietro and R. Masson, A symmetric and coercive finite volume scheme for multiphase porous media flow problems with applications in the oil industry, in Finite volumes for complex applications V. ISTE, London (2008) 35-51.

[6] L. Agelas, R. Eymard and R. Herbin, A nine-point finite volume scheme for the simulation of diffusion in heterogeneous media. C. R. Math. Acad. Sci. Paris 347 (2009) 673-676. 
[7] B. Andreianov, M. Bendahmane and K. Karlsen, A gradient reconstruction formula for finite-volume schemes and discrete duality, in Finite volumes for complex applications V. ISTE, London (2008) 161-168.

[8] B. Andreianov, M. Bendahmane, K.H. Karlsen and C. Pierre, Convergence of discrete duality finite volume schemes for the cardiac bidomain model. Netw. Heterog. Media 6 (2011) 195-240.

[9] F. Boyer and F. Hubert, Finite volume method for 2D linear and nonlinear elliptic problems with discontinuities. SIAM J. Numer. Anal. 46 (2008) 3032-3070.

[10] F. Brezzi, A. Buffa and K. Lipnikov, Mimetic finite differences for elliptic problems. ESAIM: M2AN 43 (2008) $277-295$.

[11] Y. Coudière and F. Hubert, A 3D discrete duality finite volume method for nonlinear elliptic equations. SIAM J. Sci. Comput. 33 (2011) 1739

[12] Y. Coudière, J.-P. Vila and P. Villedieu, Convergence rate of a finite volume scheme for a two-dimensional convection-diffusion problem. ESAIM: M2AN 33 (1999) 493-516.

[13] Y. Coudière, C. Pierre, O. Rousseau and R. Turpault, 2D/3D discrete duality finite volume scheme (DDFV) applied to ECG simulation. A DDFV scheme for anisotropic and heterogeneous elliptic equations, application to a bio-mathematics problem: electrocardiogram simulation, in Finite volumes for complex applications $V$. ISTE, London (2008) 313-320.

[14] Y. Coudière, C. Pierre, O. Rousseau and R. Turpault, A 2D/3D discrete duality finite volume scheme. Application to ECG simulation. Int. J. Finite 6 (2009) 24.

[15] K. Domelevo and P. Omnes, A finite volume method for the Laplace equation on almost arbitrary two-dimensional grids. ESAIM: M2AN 39 (2005) 1203-1249.

[16] J. Droniou, R. Eymard, T. Gallouët and R. Herbin, A unified approach to mimetic finite difference, hybrid finite volume and mixed finite volume methods. Math. Models Methods Appl. Sci. 20 (2010) 265-295.

[17] A. Ern and J.-L. Guermond, Theory and practice of finite elements, Applied Mathematical Sciences 159. Springer-Verlag, New York (2004).

[18] R. Eymard, T. Gallouët and P. Joly, Hybrid finite element techniques for oil recovery simulation. Comput. Methods Appl. Mech. Eng. 74 (1989) 83-98.

[19] R. Eymard, T. Gallouët and R. Herbin, Finite volume methods, in Handbook of numerical analysis, Handb. Numer. Anal. VII. North-Holland, Amsterdam (2000) 713-1020.

[20] R. Eymard, T. Gallouët and R. Herbin, A new finite volume scheme for anisotropic diffusion problems on general grids: convergence analysis. C. R. Math. Acad. Sci. Paris 344 (2007) 403-406.

[21] R. Eymard, T. Gallouët and R. Herbin, Discretisation of heterogeneous and anisotropic diffusion problems on general nonconforming meshes, SUSHI: a scheme using stabilisation and hybrid interfaces. IMA J. Numer. Anal. 30 (2010) $1009-1043$. see also http://hal.archives-ouvertes.fr/.

[22] R. Eymard, C. Guichard, R. Herbin and R. Masson, Multiphase flow in porous media using the VAG scheme, in Finite Volumes for Complex Applications VI-Problems and Persepectives, edited by J. Fort, J. Furst, J. Halama, R. Herbin and F. Hubert. Springer Proceedings in Mathematics (2011) 409-417.

[23] R. Eymard, G. Henry, R. Herbin, F. Hubert, R. Kloefkorn and G. Manzini, 3D benchmark on discretization schemes for anisotropic diffusion problem on general grids, in Finite Volumes for Complex Applications VI-Problems and Persepectives, edited by J. Fort, J. Furst, J. Halama, R. Herbin and F. Hubert. Springer Proceedings in Mathematics (2011) 95-130.

[24] I. Faille, A control volume method to solve an elliptic equation on a two-dimensional irregular mesh. Comput. Methods Appl. Mech. Eng. 100 (1992) 275-290.

[25] R. Herbin and F. Hubert, Benchmark on discretization schemes for anisotropic diffusion problems on general grids for anisotropic heterogeneous diffusion problems, in Finite Volumes for Complex Applications V, edited by R. Eymard and J.-M. Hérard. Wiley (2008) 659-692.

[26] F. Hermeline, Approximation of diffusion operators with discontinuous tensor coefficients on distorted meshes. Comput. Methods Appl. Mech. Eng. 192 (2003) 1939-1959.

[27] F. Hermeline, Approximation of 2-D and 3-D diffusion operators with variable full tensor coefficients on arbitrary meshes. Comput. Methods Appl. Mech. Eng. 196 (2007) 2497-2526.

[28] F. Hermeline, A finite volume method for approximating 3D diffusion operators on general meshes. J. Comput. Phys. 228 (2009) 5763-5786.

[29] G. Strang, Variational crimes in the finite element method, in The mathematical foundations of the finite element method with applications to partial differential equations (Proc. Sympos., Univ. Maryland, Baltimore, Md. 1972). Academic Press, New York (1972) 689-710. 